\title{
Synthesis of $11 \beta$-Perfluorohexylestradiol
}

Vangelis Agouridas, Jean-Claude Blazejewski, ${ }^{*}$ Emmanuel Magnier and Matthew D. Popkin ${ }^{\dagger}$.

SIRCOB, UMR CNRS 8086, Université de Versailles, 45 Avenue des Etats-Unis, 78035 Versailles, France

jcblaz@,chimie.uvsq.fr

\section{Table of contents :}

\begin{tabular}{|c|c|c|}
\hline Compound & Expt & Spectra \\
\hline $\begin{array}{l}11 \alpha \text {-Tridecafluorohexyl-3,17ß-dibenzyloxyestra-1,3,5(10),8(9)- } \\
\text { tetraene } 5\end{array}$ & - & S5 to $\mathrm{S7}$ \\
\hline $\begin{array}{l}\text { 11-Tridecafluorohexyl-3,17ß-dibenzyloxyestra-1,3,5(10),9(11)- } \\
\text { tetraene } 6\end{array}$ & - & S8 to $\mathrm{S10}$ \\
\hline $\begin{array}{l}\text { 11-Tridecafluorohexyl-3,17 } \beta \text {-dihydroxyestra-1,3,5(10),9(11)-tetraene } \\
7\end{array}$ & $\mathbf{S 3}$ & S11 to S13 \\
\hline 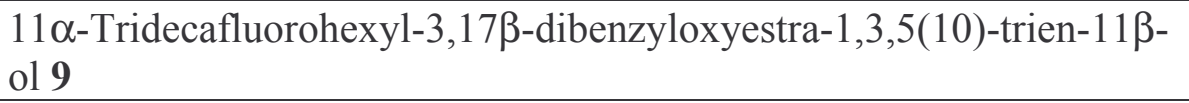 & - & S14 to S16 \\
\hline 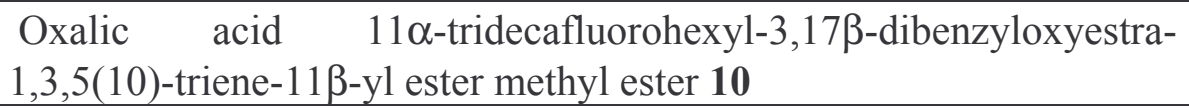 & - & S17 to S19 \\
\hline $11 \beta$-Tridecafluorohexyl-3,17 $\beta$-dibenzyloxyestra-1,3,5(10)-triene 11a & - & S20 to $\mathbf{S 2 2}$ \\
\hline $11 \alpha$-Tridecafluorohexyl-3,17ß-dibenzyloxyestra-1,3,5(10)-triene 11b & $\mathbf{S 2}$ & S23 to $\mathbf{S 2 5}$ \\
\hline $11 \alpha$-Tridecafluorohexyl-3,17 $\beta$-dihydroxyestra-1,3,5(10)-triene $\mathbf{1 2}$ & S4 & S26 to $\mathbf{S 2 8}$ \\
\hline $\begin{array}{l}\text { Oxalic acid } 11 \alpha \text {-allyl-3,17} \beta \text {-dibenzyloxyestra-1,3,5(10)-triene-11 } \beta \text {-yl } \\
\text { ester methyl ester 14a }\end{array}$ & S2 & $\mathrm{S} 29$ to $\mathrm{S30}$ \\
\hline $11 \beta$-Allyl-3,17 $\beta$-dibenzyloxyestra-1,3,5(10)-triene 15a & S3 & S31 to S32 \\
\hline $11 \alpha$-Phenyl-3,17 $\beta$-dibenzyloxyestra-1,3,5(10)-triene $\mathbf{1 5 b}$ & S3 & S33 to $\mathbf{S 3 5}$ \\
\hline $11 \beta$-Tridecafluorohexyl-3,17 $\beta$-dihydroxyestra-1,3,5(10)-triene $\mathbf{1 e}$ & - & S35 to $\mathbf{S 4 1}$ \\
\hline Spectra of compounds 5 and $7: \Delta 8(9)$ or $\Delta 9(11) ?$ & & S42 \\
\hline
\end{tabular}




\section{Oxalic acid 11 $\alpha$-allyl-3,17 $\beta$-dibenzyloxyestra-1,3,5(10)-triene-11 $\beta$-yl ester methyl ester} (14a)

89\% yield; ${ }^{1} \mathrm{H}$ NMR $\left(300 \mathrm{MHz}, \mathrm{CDCl}_{3}\right) \delta 0.85(\mathrm{~s}, 3 \mathrm{H}), 1.09-1.66(\mathrm{~m}, 7 \mathrm{H}), 1.80-1.99(\mathrm{~m}$, 2H), $2.19(\mathrm{~d}, J=11.1 \mathrm{~Hz}, 2 \mathrm{H}), 2.47-2.58(\mathrm{~m}, 2 \mathrm{H}), 3.11-3.19(\mathrm{~m}, 2 \mathrm{H}), 3.31(\mathrm{dd}, J=13.8$, $8.4 \mathrm{~Hz}, 1 \mathrm{H}), 3.39$ (t, J = 7.8 Hz, 1H), 3.73 (s, 3H), $4.46(\mathrm{~s}, 2 \mathrm{H}), 4.88-4.95$ (m, 4H), 5.51 (m, $1 \mathrm{H}), 6.69-6.72(\mathrm{~m}, 2 \mathrm{H}), 7.14-7.48(\mathrm{~m}, 11 \mathrm{H}) ;{ }^{13} \mathrm{C} \mathrm{NMR}\left(75 \mathrm{MHz}, \mathrm{CDCl}_{3}\right) \delta$ 12.6, 23.9, 25.7, 27.6, 28.7, 33.3, 42.9, 43.2, 43.4, 48.4, 51.0, 53.3, 69.9, 71.7, 88.7, 90.9, 111.3, 114.1, $119.5,127.4,127.5,127.7,127.9,128.3,128.6,129.5,133.2,137.3,139.0,142.5,156.8$, 157.4, 158.9; MS pos. ESI (m/z): $633\left(\mathrm{M}+\mathrm{K}^{+}\right), 617\left(\mathrm{M}+\mathrm{Na}^{+}\right), 491\left(\mathrm{MH}-\mathrm{MeOCOCO}_{2} \mathrm{H}\right)$; $[\alpha]^{25}-43.3$ (c 0.85 , dichloromethane).

Oxalic acid $11 \alpha$-phenyl-3,17 $\beta$-dibenzyloxyestra-1,3,5(10)-triene-11 $\beta$-yl ester methyl ester (14b)

This particular oxalate, being too unstable could not be isolated, even for recording an NMR spectrum, and was used directly for the reduction experiment after evaporation of the solvent.

\section{$11 \alpha$-Tridecafluorohexyl-3,17 $\beta$-dibenzyloxyestra-1,3,5(10)-triene (11b).}

${ }^{1} \mathrm{H}$ NMR (300 MHz, $\left.\mathrm{CDCl}_{3}\right) \delta 0.70(\mathrm{~s}, 3 \mathrm{H}), 1.14-1.75(\mathrm{~m}, 10 \mathrm{H}), 2.00(\mathrm{~m}, 1 \mathrm{H}), 2.22(\mathrm{~m}$, 1H), $2.36(\mathrm{~m}, 1 \mathrm{H}), 2.59-2.75(\mathrm{~m}, 2 \mathrm{H}), 3.32(\mathrm{~m}, 1 \mathrm{H}), 3.42(\mathrm{t}, J=8.4 \mathrm{~Hz}, 1 \mathrm{H}), 4.47$ (AB system, $J=12 \mathrm{~Hz}, 2 \mathrm{H}), 4.96(\mathrm{~s}, 2 \mathrm{H}), 6.71-6.74(\mathrm{~m}, 2 \mathrm{H}), 6.96(\mathrm{dd}, J=8.7,7.2 \mathrm{~Hz}, 1 \mathrm{H}), 7.18$ $-7.37(\mathrm{~m}, 10 \mathrm{H}) ;{ }^{13} \mathrm{C} \mathrm{NMR}\left(75 \mathrm{MHz}, \mathrm{CDCl}_{3}\right) \delta 11.7,23.6,25.9,26.9,27.7,35.8\left(\mathrm{dd}, \mathrm{J}_{\mathrm{CF}}=21\right.$ Hz, 19 Hz), 36.1, 38.0, 40.3, 42.3, 49.4, 70.0, 71.8, 87.7, 110.9, 114.4, 123.1, 127.4, 127.5, $127.9,128.3,128.6,135.9,137.3,139.0,139.5,157.0 ;{ }^{19} \mathrm{~F} \mathrm{NMR}\left(188 \mathrm{MHz}, \mathrm{CDCl}_{3}\right) \delta-81.2$ (t, $J=10 \mathrm{~Hz}, 3 \mathrm{~F}),-106.6$ and -118.8 (AB system, $J=280 \mathrm{~Hz}, 2 \mathrm{~F}),-120.0(\mathrm{~m}, 2 \mathrm{~F}),-122.0--$ $123.5(\mathrm{~m}, 4 \mathrm{~F}),-126.6(\mathrm{~m}, 2 \mathrm{~F})$; MS pos. ESI $(\mathrm{m} / \mathrm{z}): 819\left(\mathrm{M}+\mathrm{K}^{+}\right), 793\left(\mathrm{M}+\mathrm{Na}^{+}\right) ;[\alpha]^{25}{ }_{\mathrm{D}}-97.6$ (c 0.38 , dichloromethane). 


\section{1 $\beta$-Allyl-3,17 $\beta$-dibenzyloxyestra-1,3,5(10)-triene (15a) (lit. ${ }^{13}$ )}

Mp 100-103 ${ }^{\circ} \mathrm{C} ;{ }^{1} \mathrm{H}$ NMR (300 MHz, $\left.\mathrm{CDCl}_{3}\right) \delta 1.03(\mathrm{~s}, 3 \mathrm{H}), 1.26-1.65(\mathrm{~m}, 7 \mathrm{H}), 1.85-$ $2.08(\mathrm{~m}, 4 \mathrm{H}), 2.34(\mathrm{~d}, J=13.4 \mathrm{~Hz}, 1 \mathrm{H}), 2.51-2.58(\mathrm{~m}, 2 \mathrm{H}), 2.70-2.82(\mathrm{~m}, 2 \mathrm{H}), 3.48(\mathrm{t}, J=$ $8.1 \mathrm{~Hz}, 1 \mathrm{H}), 4.57(\mathrm{~s}, 2 \mathrm{H}), 4.95-5.02(\mathrm{~m}, 4 \mathrm{H}), 5.82(\mathrm{~m}, 1 \mathrm{H}), 6.69(\mathrm{dd}, J=8.4,2.4 \mathrm{~Hz}, 1 \mathrm{H})$, $7.08(\mathrm{~d}, J=8.4 \mathrm{~Hz}, 1 \mathrm{H}), 7.25-7.44(\mathrm{~m}, 10 \mathrm{H}) ;{ }^{13} \mathrm{C} \mathrm{NMR}\left(75 \mathrm{MHz}, \mathrm{CDCl}_{3}\right) \delta 15.7,23.3,27.2$, $28.2,30.6,33.3,34.4,36.9,39.8,43.9,49.3,52.2,70.0,71.8,89.8,113.0,114.9,115.9,127.5$, 127.6, 127.8, 128.0, 128.4, 128.7, 130.5, 137.5, 139.0, 139.1, 139.5, 156.6; $\mathrm{CIMS} \mathrm{NH}_{3}(\mathrm{~m} / \mathrm{z})$ : $510\left(\mathrm{M}+\mathrm{NH}_{4}^{+}\right), 493\left(\mathrm{M}+\mathrm{H}^{+}\right) ;[\alpha]^{25} 40.4(\mathrm{c} 0.23$, diethylether).

\section{1 $\alpha$-Phenyl-3,17 $\beta$-dibenzyloxyestra-1,3,5(10)-triene (15b).}

${ }^{1} \mathrm{H}$ NMR $\left(300 \mathrm{MHz}, \mathrm{CDCl}_{3}\right) \delta 0.94(\mathrm{~s}, 3 \mathrm{H}, \mathrm{H} 18), 1.15-1.82(\mathrm{~m}, 8 \mathrm{H}), 1.96-2.08(\mathrm{~m}, 3 \mathrm{H})$, $2.18-2.27(\mathrm{~m}, 1 \mathrm{H}), 2.67-2.82(\mathrm{~m}, 3 \mathrm{H}), 3.51(\mathrm{t}, J=8.7 \mathrm{~Hz}, 1 \mathrm{H}), 4.44-4.54$ (AB system, $J$ $=12.1 \mathrm{~Hz}, 2 \mathrm{H}), 4.85(\mathrm{~s}, 2 \mathrm{H}), 6.21(\mathrm{dd}, J=2.7,8.7 \mathrm{~Hz}, 1 \mathrm{H}), 6.51(\mathrm{~d}, J=8.7 \mathrm{~Hz}, 1 \mathrm{H}), 6.56(\mathrm{~d}$, $J=2.7 \mathrm{~Hz}, 1 \mathrm{H}), 7.16-7.28(\mathrm{~m}, 15 \mathrm{H}) ;{ }^{13} \mathrm{C} \mathrm{NMR}\left(75 \mathrm{MHz}, \mathrm{CDCl}_{3}\right) \delta 11.6,24.3,26.6,28.2$, $29.2,29.5,39.3,42.1,48.0,48.8,69.7,71.8,88.4,111.5,113.7,127.4,127.5,127.9,128.3$ $128.4,128.5,128.6,129.6,132.1,133.2,137.2,139.1,139.2,145.4,156.9$. MS pos. ESI (m/z): $567\left[\mathrm{M}+\mathrm{K}^{+}\right], 551\left[\mathrm{M}+\mathrm{Na}^{+}\right]$; HRMS obsd 528.3032, calcd $528.3028\left(\mathrm{C}_{38} \mathrm{H}_{40} \mathrm{O}_{2}\right)$.

\section{1-Tridecafluorohexyl-3,17 $\beta$-dihydroxyestra-1,3,5(10),9(11)-tetraene (7).}

91\% yield; white solid: mp 171-172 ${ }^{\circ} \mathrm{C} ;{ }^{1} \mathrm{H}$ NMR (300 MHz, $\left.\mathrm{CD}_{3} \mathrm{OD}\right) \delta 0.71(\mathrm{~s}, 3 \mathrm{H}), 1.19$ $1.55(\mathrm{~m}, 5 \mathrm{H}), 1.70-1.87(\mathrm{~m}, 2 \mathrm{H}), 1.94-2.05(\mathrm{~m}, 3 \mathrm{H}), 2.33-2.55(\mathrm{~m}, 3 \mathrm{H}), 3.21(\mathrm{~s}, 1 \mathrm{H}), 3.68$ $(\mathrm{t}, J=8.8 \mathrm{~Hz}, 1 \mathrm{H}), 6.42-6.46(\mathrm{~m}, 2 \mathrm{H}), 6.90(\mathrm{~m}, 1 \mathrm{H}) ;{ }^{13} \mathrm{C} \mathrm{NMR}\left(75 \mathrm{MHz}, \mathrm{CD}_{3} \mathrm{OD}\right) \delta 10.6$, $25.2,28.0,29.0,30.8,40.2,42.7,42.8,48.7,82.2,112.9,114.3,119.3,129.2,130.7,142.0$ 149.4, 159.1; ${ }^{19} \mathrm{~F}$ NMR (188 MHz, $\left.\mathrm{CD}_{3} \mathrm{OD}\right) \delta-80.0(\mathrm{t}, J=9.1 \mathrm{~Hz}, 3 \mathrm{~F}),-96.8$ and -100.4 (AB system, $J=270 \mathrm{~Hz}, 2 \mathrm{~F}),-115.0(\mathrm{~m}, 2 \mathrm{~F}),-119.3(\mathrm{~m}, 2 \mathrm{~F}),-120.3(\mathrm{~m}, 2 \mathrm{~F}),-123.9(\mathrm{~m}, 2 \mathrm{~F})$; MS neg. ESI (m/z): $633\left(\mathrm{M}+\right.$ formate), $623\left(\mathrm{M}+\mathrm{Cl}^{-}\right), 587(\mathrm{M}-1) ;[\alpha]^{25}{ }_{\mathrm{D}} 127.3(\mathrm{c} 0.6$, methanol$\left.d_{4}\right)$; HRMS obsd 588.0790, calcd $588.1334\left(\mathrm{C}_{24} \mathrm{H}_{21} \mathrm{O}_{2} \mathrm{~F}_{13}\right)$. 


\section{$11 \alpha$-Tridecafluorohexyl-3,17 $\beta$-dihydroxyestra-1,3,5(10)-triene (12).}

94\% yield : mp 243-245 ${ }^{\circ} \mathrm{C} ;{ }^{1} \mathrm{H}$ NMR (300 MHz, methanol- $\left.d_{4}\right) \delta 0.58(\mathrm{~s}, 3 \mathrm{H}), 0.99-1.50$ (m, 8H), $1.56-1.69(\mathrm{~m}, 2 \mathrm{H}), 1.95(\mathrm{~m}, 1 \mathrm{H}), 2.15(\mathrm{~d}, J=12.6 \mathrm{~Hz}, 1 \mathrm{H}), 2.31(\mathrm{t}, J=10.2 \mathrm{~Hz}$ 1H), $2.51-2.67(\mathrm{~m}, 2 \mathrm{H}), 3.21(\mathrm{~s}, 1 \mathrm{H}), 3.32(\mathrm{~m}, 1 \mathrm{H}), 3.59(\mathrm{t}, J=8.7 \mathrm{~Hz}, 1 \mathrm{H}), 6.46-6.49(\mathrm{~m}$, 2H), $6.82(\mathrm{dd}, J=9.0,6.3 \mathrm{~Hz}, 1 \mathrm{H}) ;{ }^{13} \mathrm{C} \mathrm{NMR}\left(75 \mathrm{MHz}\right.$, methanol- $\left.d_{4}\right) \delta 11.4,24.4,27.1,27.7$, 30.6, 36.9, $37.2\left(\mathrm{t}, J_{C F}=18 \mathrm{~Hz}\right), 39.2,42.2,43.4,50.5,82.0,112.7,115.6,124.1,135.6$, 140.6, 156.3; ${ }^{19} \mathrm{~F}$ NMR (188 MHz, methanol- $\left.d_{4}\right) \delta-78.9(\mathrm{t}, J=10.2 \mathrm{~Hz}, 3 \mathrm{~F}),-103.7$ and 115.1 (AB system, $J=281 \mathrm{~Hz}, 2 \mathrm{~F}),-117.0$ (broad s, 2F), -119.0 - -120.5 (m, 4F), -123.8 (m, 2F); IR ( $\left.\mathrm{cm}^{-1}, \mathrm{KBr}\right): 3298,2944,2913,1234$; MS neg. ESI (m/z): 635 (M + formate), 625.2 $\left(\mathrm{M}+\mathrm{Cl}^{-}\right), 589(\mathrm{M}-1) ;[\alpha]_{\mathrm{D}}^{25}-96.8\left(\mathrm{c} 0.6\right.$, methanol- $\left.d_{4}\right)$. 


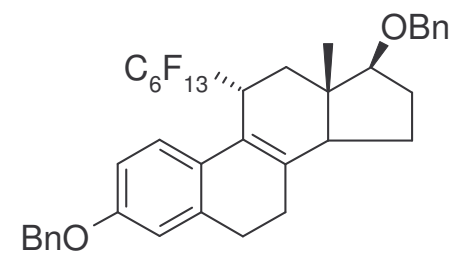

$11 \alpha$-Tridecafluorohexyl-3,17 $\beta$-dibenzyloxyestra-

$$
\begin{gathered}
\text { 1,3,5(10),8(9)-tetraene (5) } \\
\mathrm{C}_{38} \mathrm{H}_{35} \mathrm{~F}_{13} \mathrm{O}_{2} \\
\mathrm{M}=768 \mathrm{~g} \cdot \mathrm{mol}^{-1}
\end{gathered}
$$

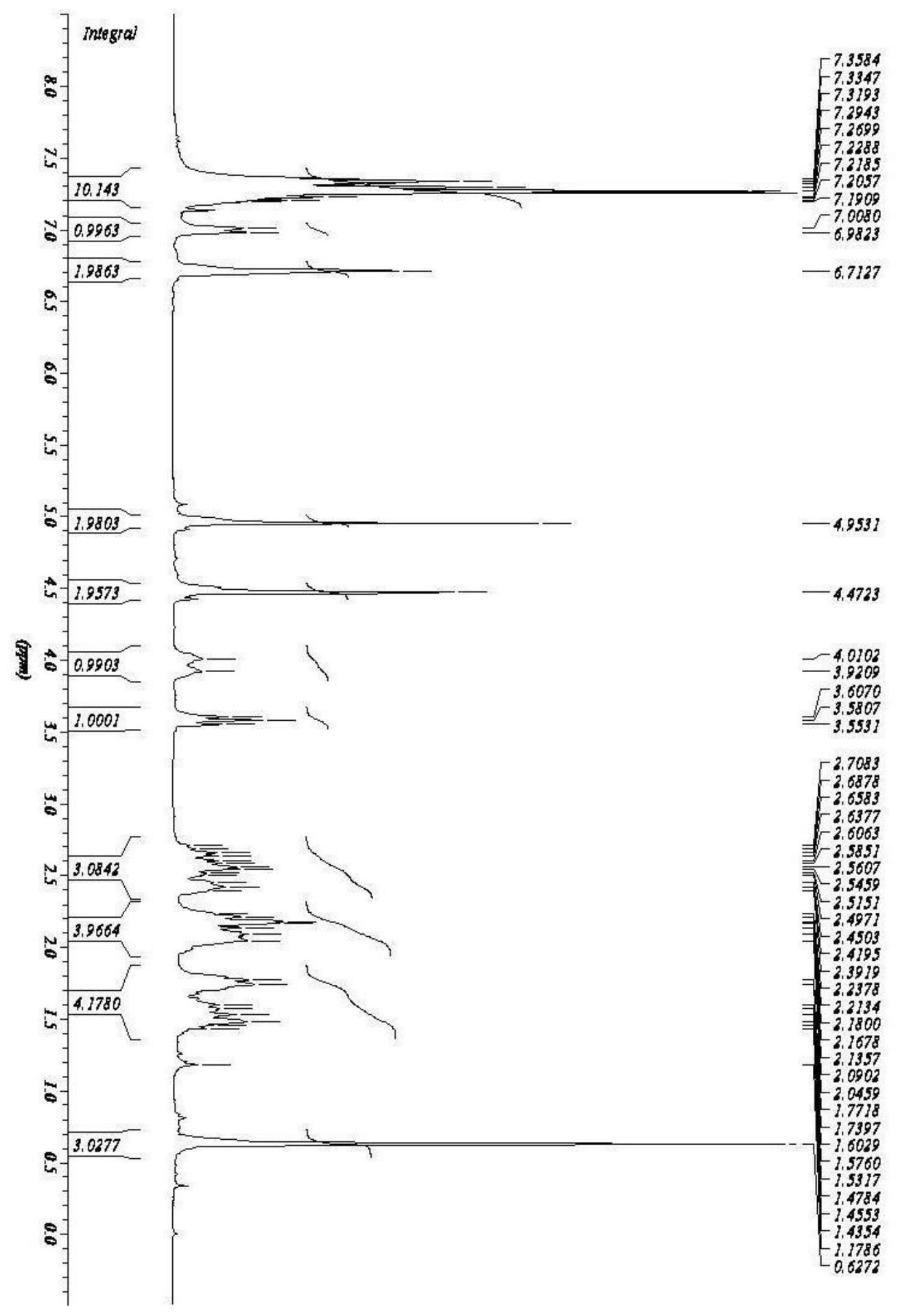



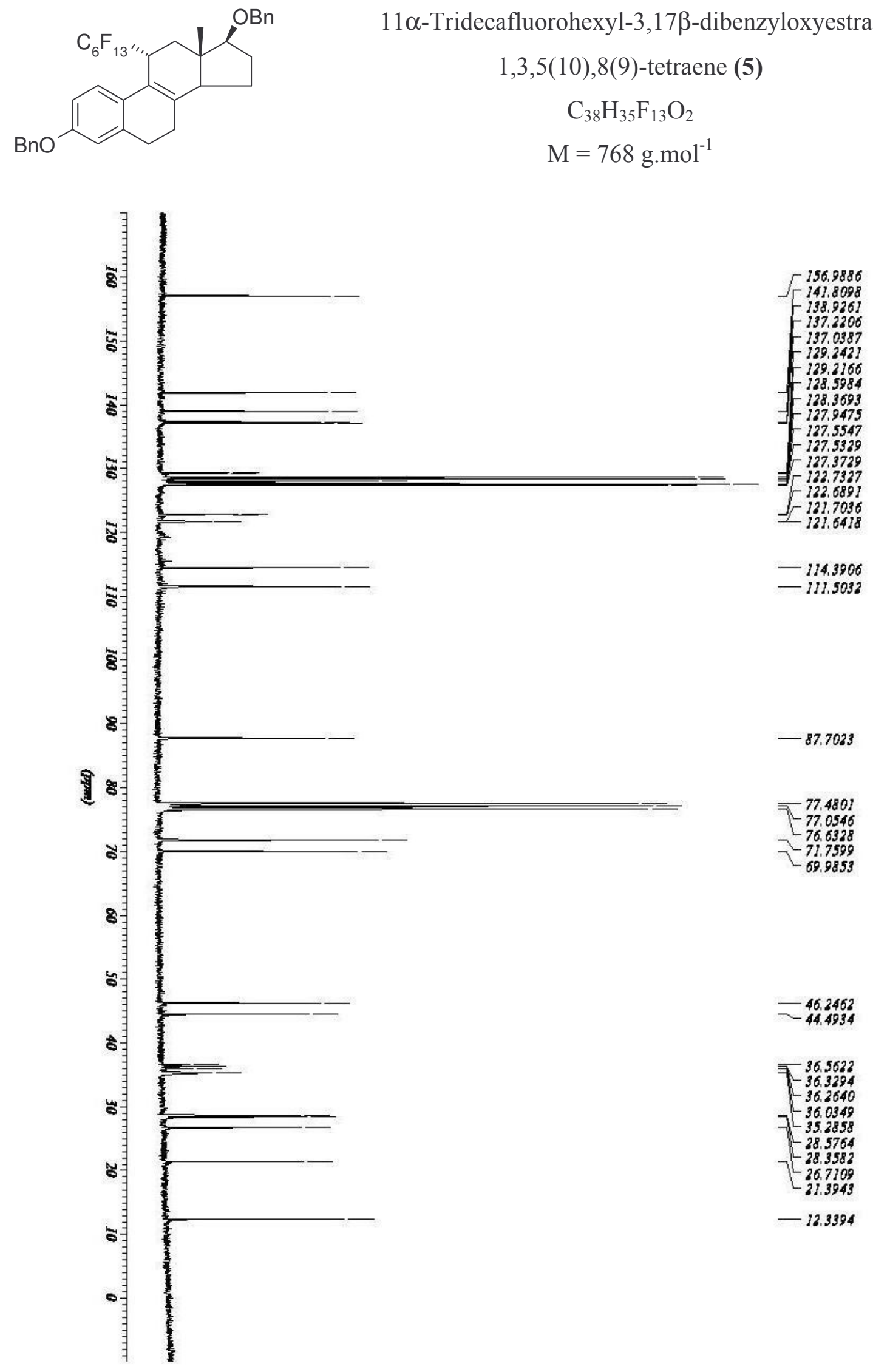

$11 \alpha$-Tridecafluorohexyl-3,17 $\beta$-dibenzyloxyestra1,3,5(10),8(9)-tetraene (5)

$$
\mathrm{C}_{38} \mathrm{H}_{35} \mathrm{~F}_{13} \mathrm{O}_{2}
$$$$
\mathrm{M}=768 \mathrm{~g} \cdot \mathrm{mol}^{-1}
$$ 
<smiles>C[C@@H](Cc1ccccc1)C1=C2CCc3cc(OCc4ccccc4)ccc3C2=C[C@H]1C(F)(F)F</smiles>

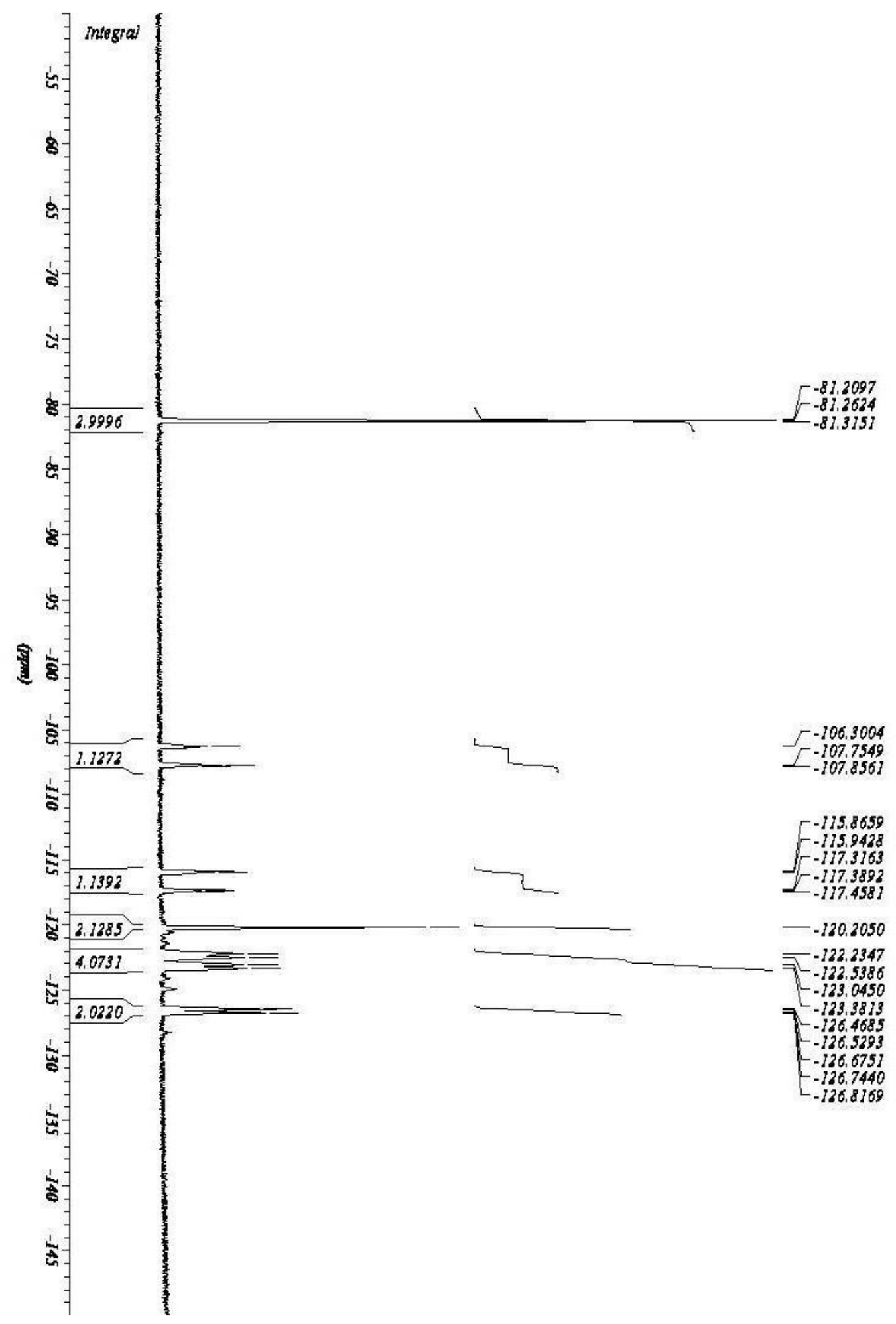

$11 \alpha$-Tridecafluorohexyl-3,17 $\beta$-dibenzyloxyestra-

$$
\begin{gathered}
\text { 1,3,5(10),8(9)-tetraene (5) } \\
\mathrm{C}_{38} \mathrm{H}_{35} \mathrm{~F}_{13} \mathrm{O}_{2} \\
\mathrm{M}=768 \mathrm{~g} \cdot \mathrm{mol}^{-1}
\end{gathered}
$$


<smiles>CC1(C(F)(F)F)CC(C(F)(F)F)=C2c3ccc(OCc4ccccc4)cc3CCC2C1Cc1ccccc1</smiles>

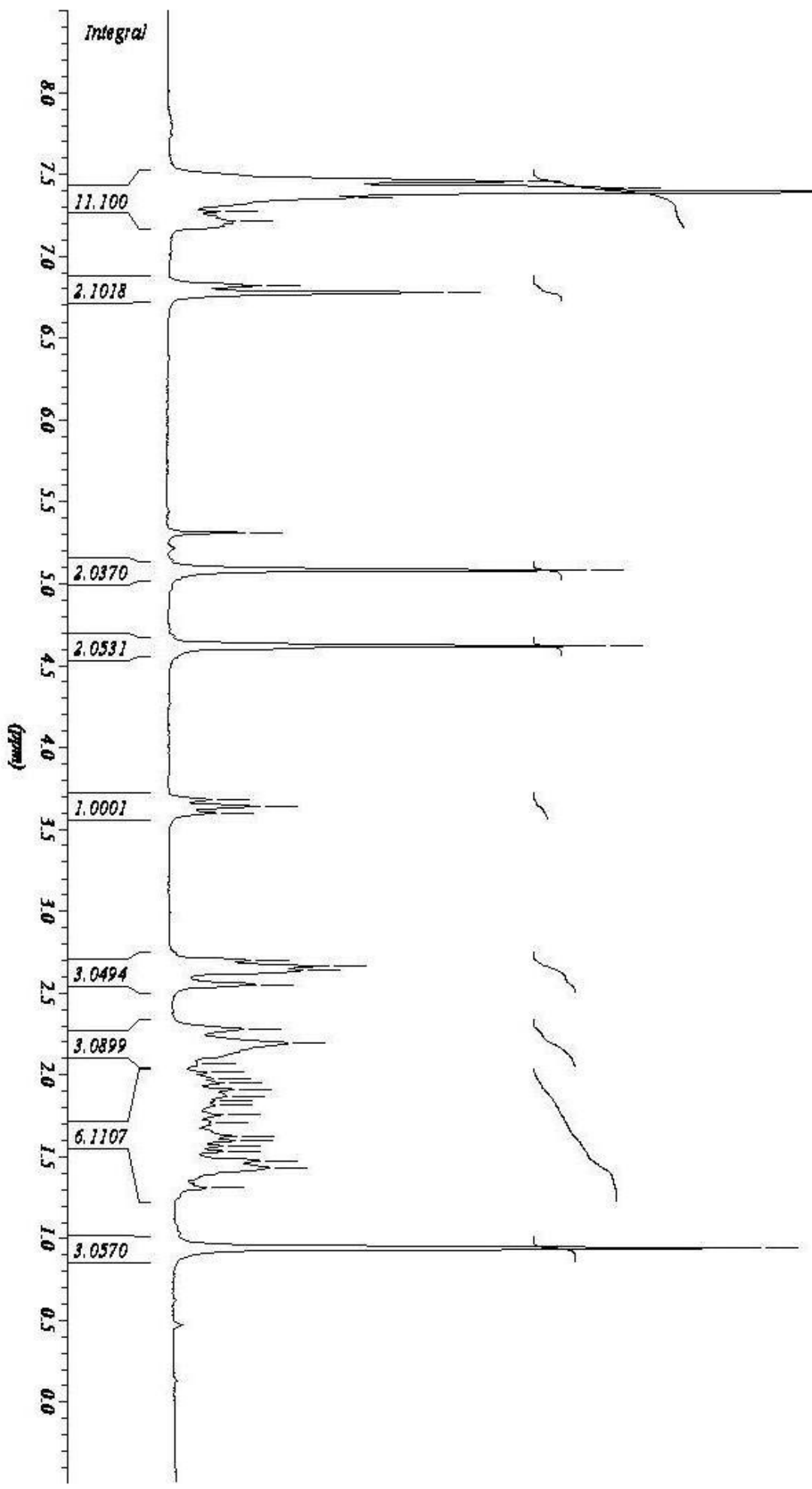

11-Tridecafluorohexyl-3,17 $\beta$-dibenzyloxyestra-

$$
\text { 1,3,5(10),9(11)-tetraene (6) }
$$$$
\mathrm{C}_{38} \mathrm{H}_{35} \mathrm{~F}_{13} \mathrm{O}_{2}
$$$$
\mathrm{M}=768 \mathrm{~g} \cdot \mathrm{mol}^{-1}
$$

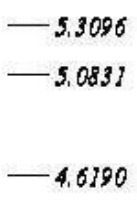

3.6800
-3.6388
3.5977

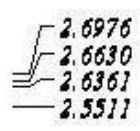

$\begin{array}{r}-2.2769 \\ -\quad 3.1907 \\ \hline\end{array}$

3.1907

2,0125

1.9746

$-1,9077$

$+1,8655$

$-1.8370$

$-1.8134$

$-17179$

$-1,6263$

$-1.5956$

$-1.5627$

$-1.5314$

$-1,4744$

$-1,4283$

$-0,9396$ 
<smiles>CC1(C(F)(F)F)CC(Cc2ccccc2)=C2c3ccc(OCc4ccccc4)cc3CCC2C1COc1ccccc1</smiles>

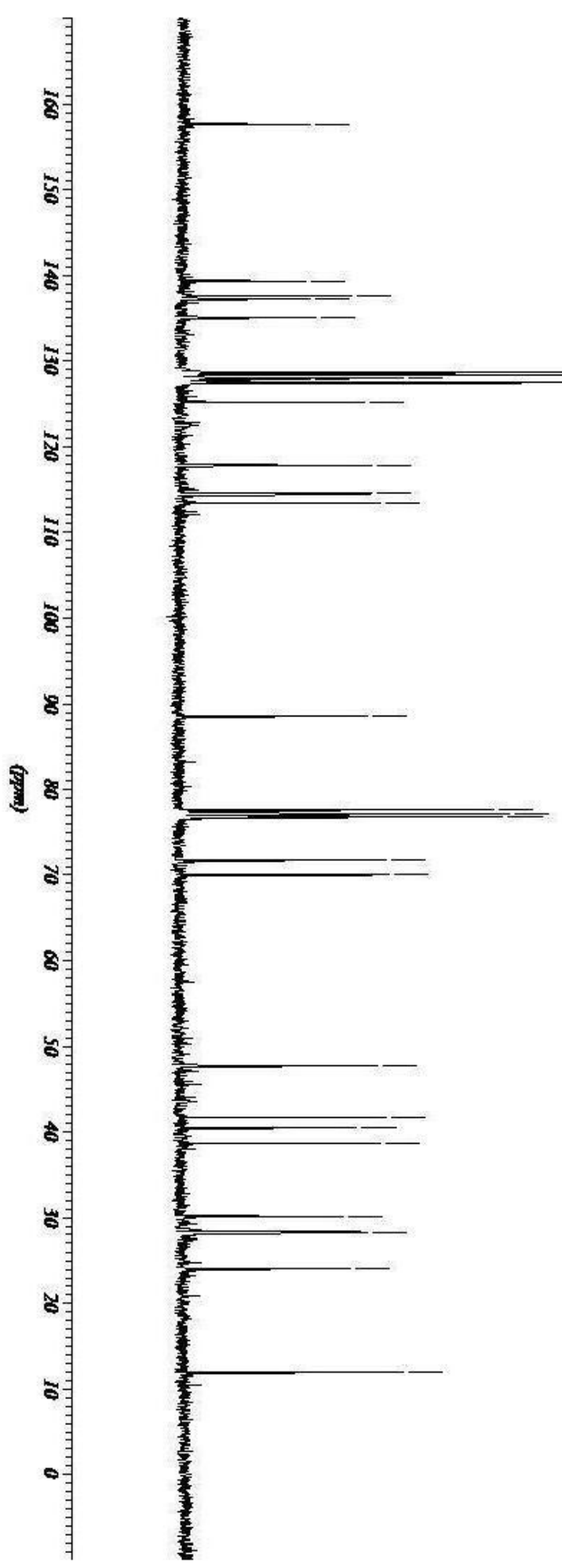

11-Tridecafluorohexyl-3,17 $\beta$-dibenzyloxyestra-

$$
\begin{gathered}
\text { 1,3,5(10),9(11)-tetraene (6) } \\
\mathrm{C}_{38} \mathrm{H}_{35} \mathrm{~F}_{13} \mathrm{O}_{2} \\
\mathrm{M}=768 \mathrm{~g} \cdot \mathrm{mol}^{-1}
\end{gathered}
$$


<smiles>CC(F)(F)C1=C2c3ccc(OCc4ccccc4)cc3CCC2C2CC[C@@H](O)[C@]12C</smiles>

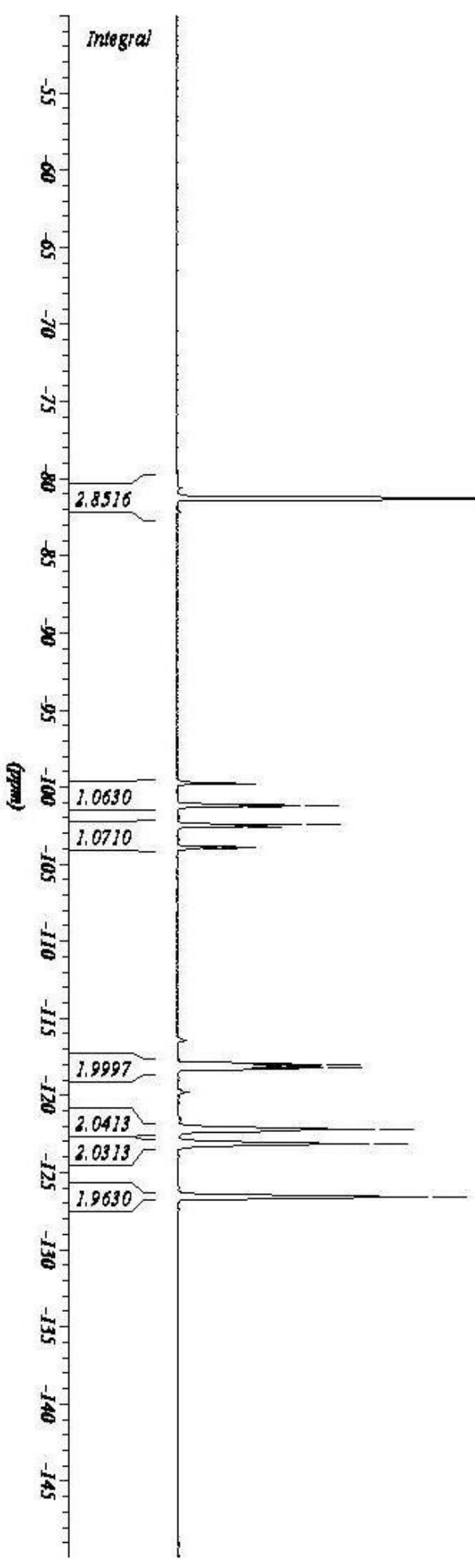

11-Tridecafluorohexyl-3,17 $\beta$-dibenzyloxyestra-

$$
\text { 1,3,5(10),9(11)-tetraene (6) }
$$$$
\mathrm{C}_{38} \mathrm{H}_{35} \mathrm{~F}_{13} \mathrm{O}_{2}
$$$$
\mathrm{M}=768 \mathrm{~g} \cdot \mathrm{mol}^{-1}
$$ 
<smiles>C[C@]12CC(C(F)(F)F)=C3c4ccc(O)cc4CCC3C1CC[C@@H]2O</smiles>

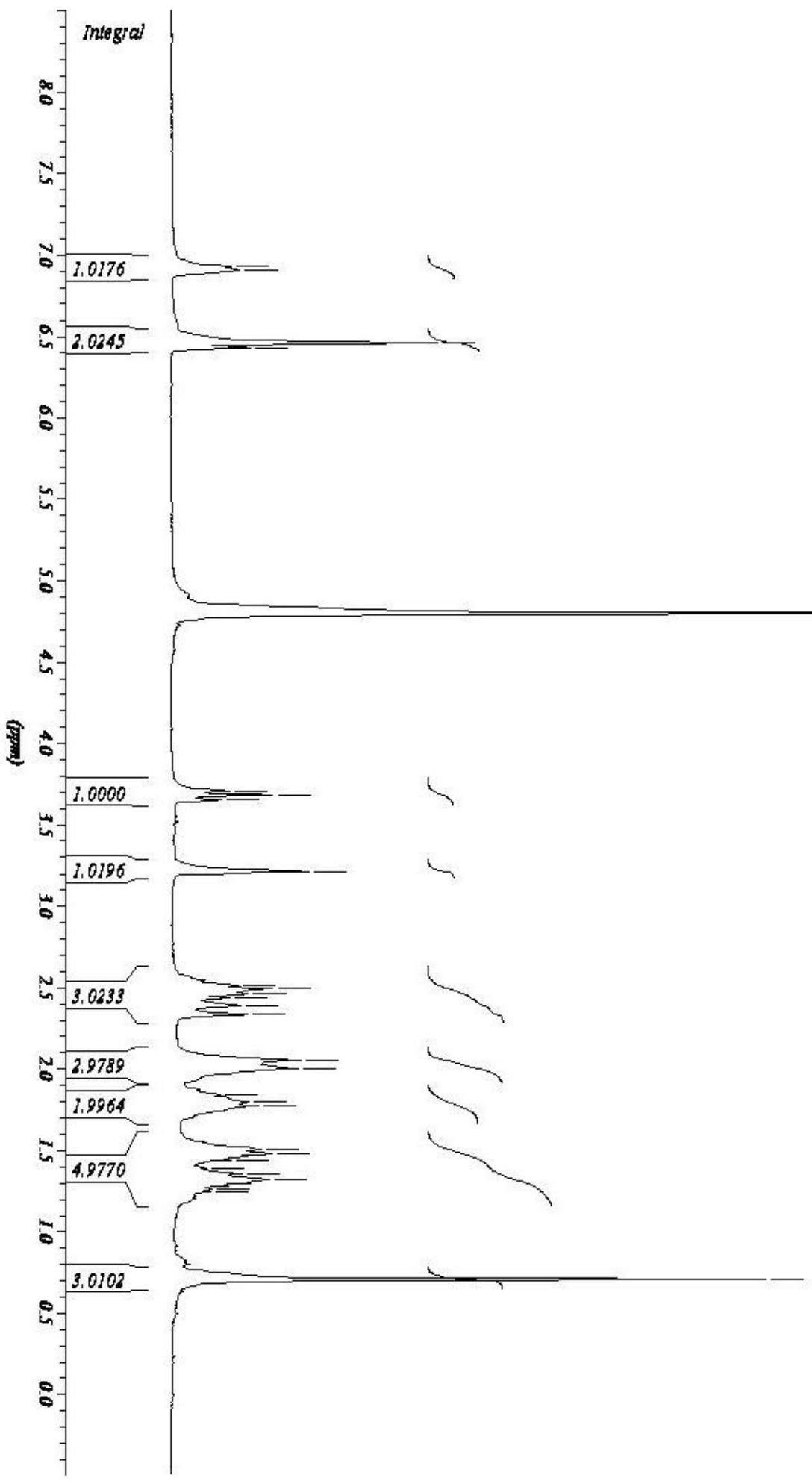

11-Tridecafluorohexyl-3,17 $\beta$-dihydroxyestra-

$$
\begin{gathered}
\text { 1,3,5(10),9(11)-tetraene (7) } \\
\mathrm{C}_{24} \mathrm{H}_{21} \mathrm{~F}_{13} \mathrm{O}_{2} \\
\mathrm{M}=588 \mathrm{~g} \cdot \mathrm{mol}^{-1}
\end{gathered}
$$
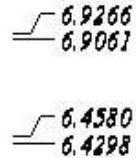

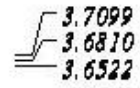

$-3.2131$

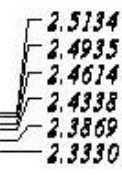

$-2.0518$

$=-1.8413$

17976

$-1.7733$

(-1.4818

$1,44,14$

$-1,3913$

-1.3547
-1.3194
-1.2610

$-1.2610$

1.2443
0.7057 
<smiles>C[C@]12CC(C(F)(F)F)=C3c4ccc(O)cc4CCC3C1CC[C@@H]2O</smiles>

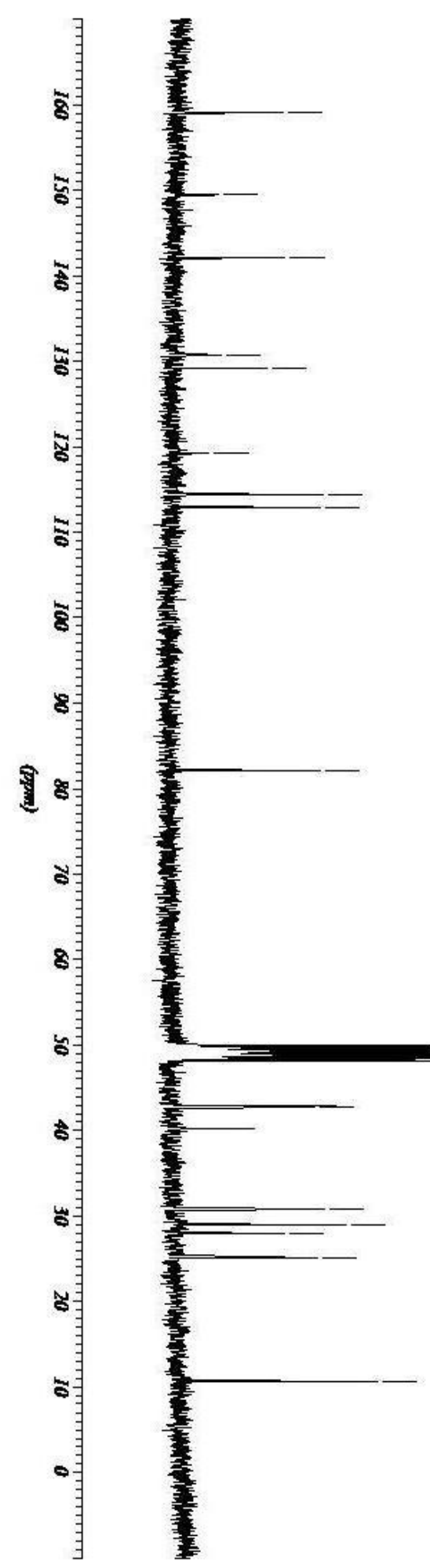

11-Tridecafluorohexyl-3,17ß-dihydroxyestra-

$$
\begin{gathered}
\text { 1,3,5(10),9(11)-tetraene (7) } \\
\mathrm{C}_{24} \mathrm{H}_{21} \mathrm{~F}_{13} \mathrm{O}_{2} \\
\mathrm{M}=588 \mathrm{~g} \cdot \mathrm{mol}^{-1}
\end{gathered}
$$

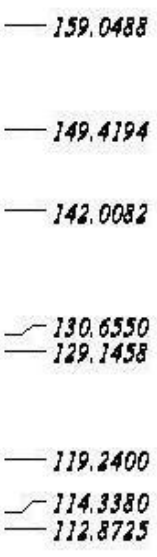

$-82.1149$

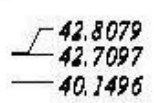

$-10.5930$ 
<smiles>CC(F)(F)C1=C2c3ccc(O)cc3CCC2C2CCC(O)C2(C)C1</smiles>

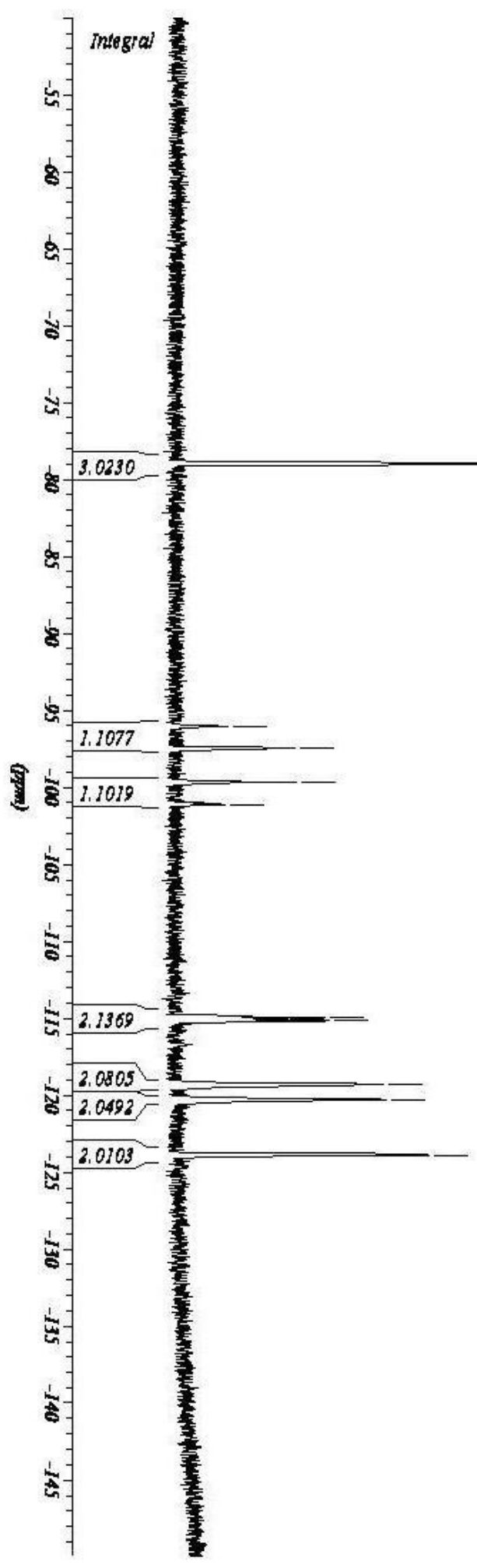

11-Tridecafluorohexyl-3,17 $\beta$-dihydroxyestra-

$$
\begin{gathered}
\text { 1,3,5(10),9(11)-tetraene (7) } \\
\mathrm{C}_{24} \mathrm{H}_{21} \mathrm{~F}_{13} \mathrm{O}_{2} \\
\mathrm{M}=588 \mathrm{~g} \cdot \mathrm{mol}^{-1}
\end{gathered}
$$
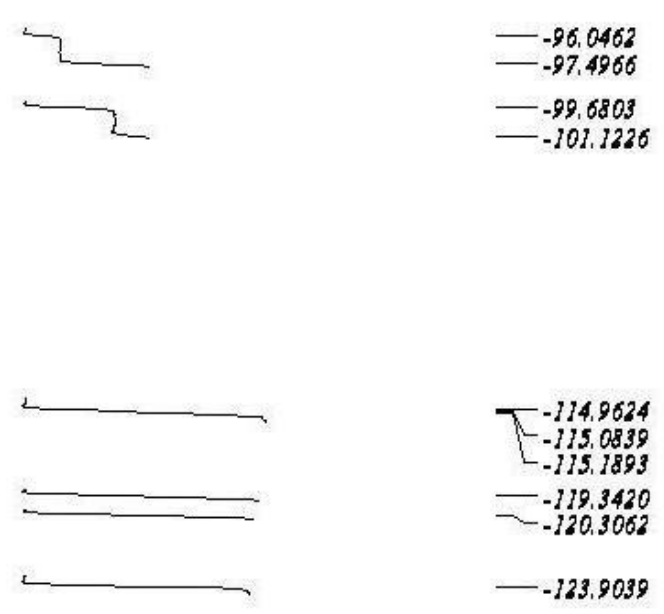


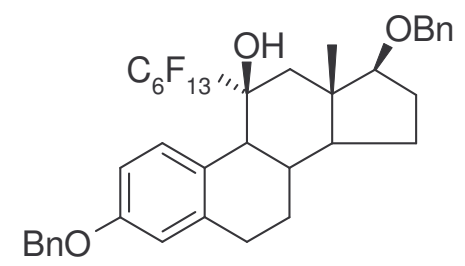

$11 \alpha$-Tridecafluorohexyl-3,17 $\beta$-dibenzyloxyestra-1,3,5(10)-

$$
\begin{gathered}
\text { trien-11ß-ol (9) } \\
\mathrm{C}_{38} \mathrm{H}_{37} \mathrm{~F}_{13} \mathrm{O}_{3} \\
\mathrm{M}=786 \text { g.mol }{ }^{-1}
\end{gathered}
$$

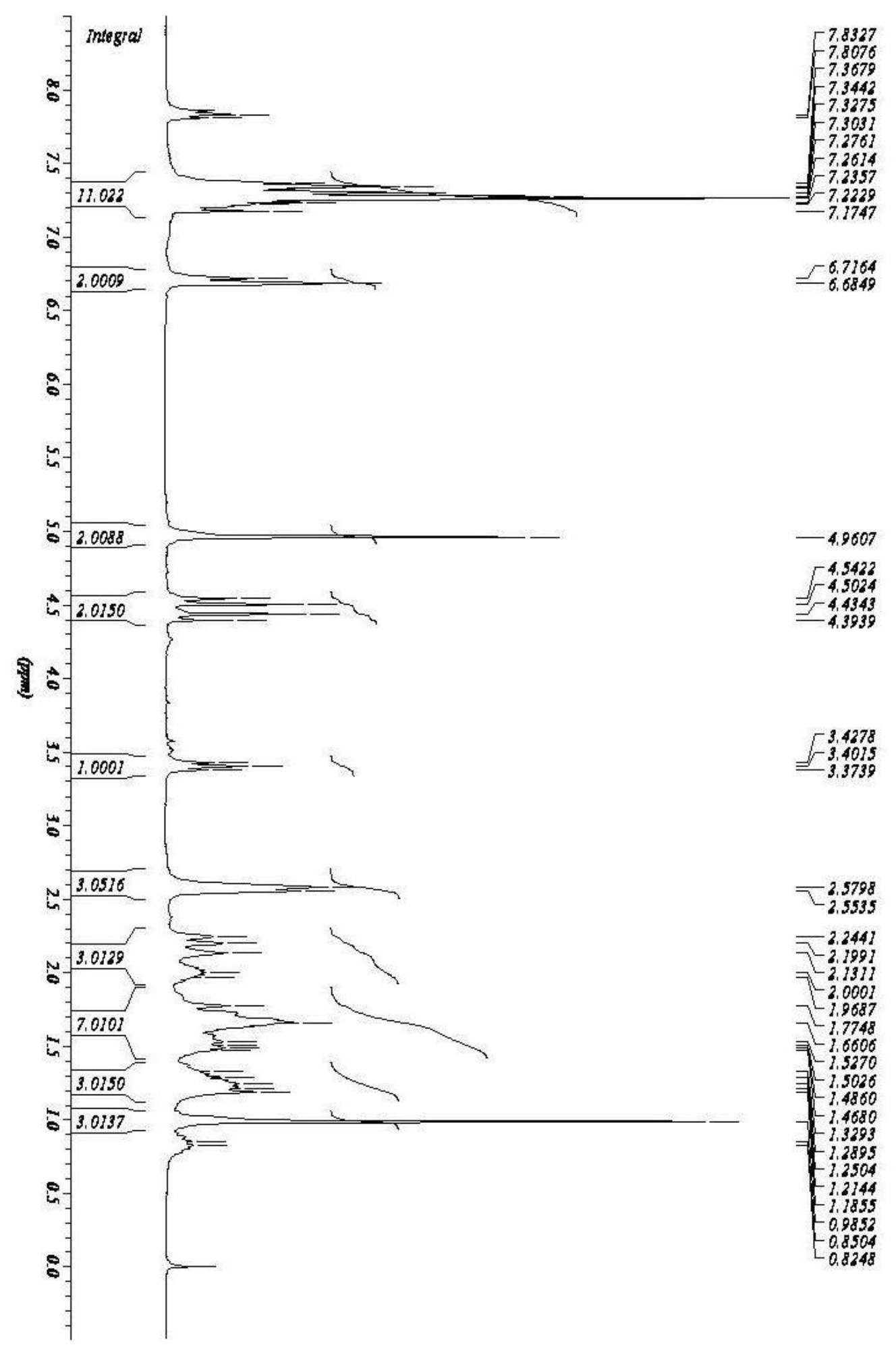




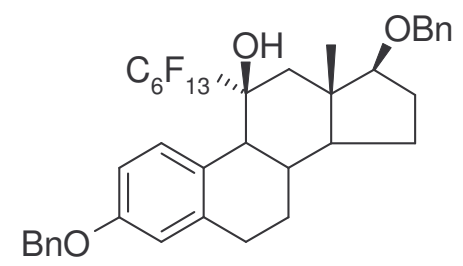

$11 \alpha$-Tridecafluorohexyl-3,17 $\beta$-dibenzyloxyestra-1,3,5(10)-

$$
\begin{gathered}
\text { trien-11ß-ol (9) } \\
\mathrm{C}_{38} \mathrm{H}_{37} \mathrm{~F}_{13} \mathrm{O}_{3} \\
\mathrm{M}=786 \mathrm{~g} \cdot \mathrm{mol}^{-1}
\end{gathered}
$$

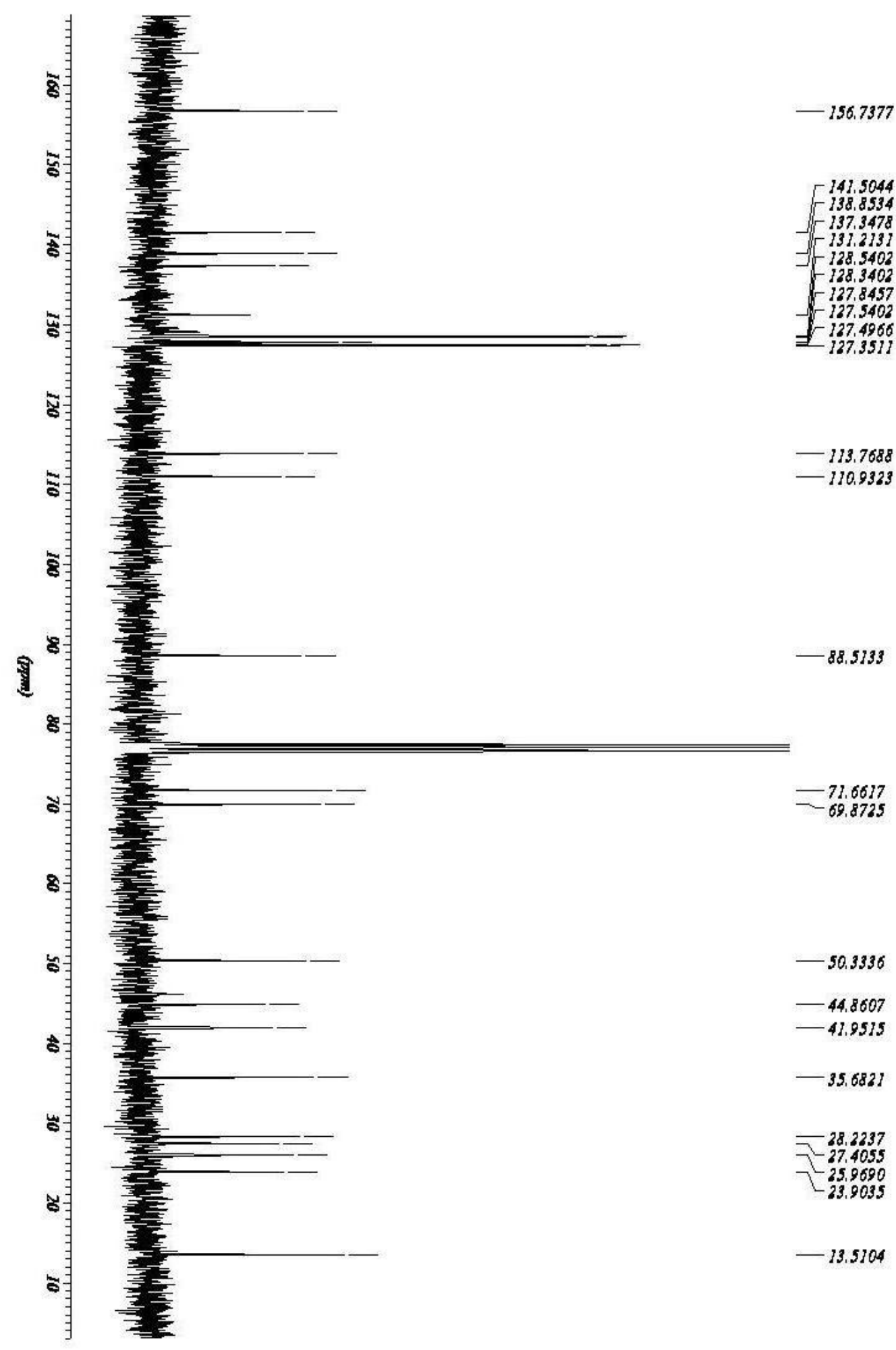




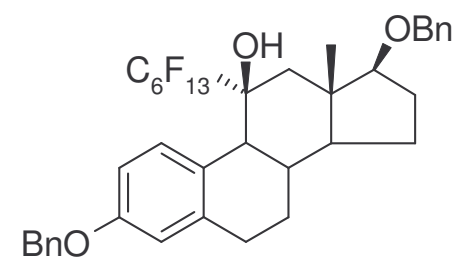

$11 \alpha$-Tridecafluorohexyl-3,17 $\beta$-dibenzyloxyestra-1,3,5(10)-

$$
\begin{gathered}
\text { trien-11 } \beta \text {-ol (9) } \\
\mathrm{C}_{38} \mathrm{H}_{37} \mathrm{~F}_{13} \mathrm{O}_{3} \\
\mathrm{M}=786 \text { g.mol }
\end{gathered}
$$

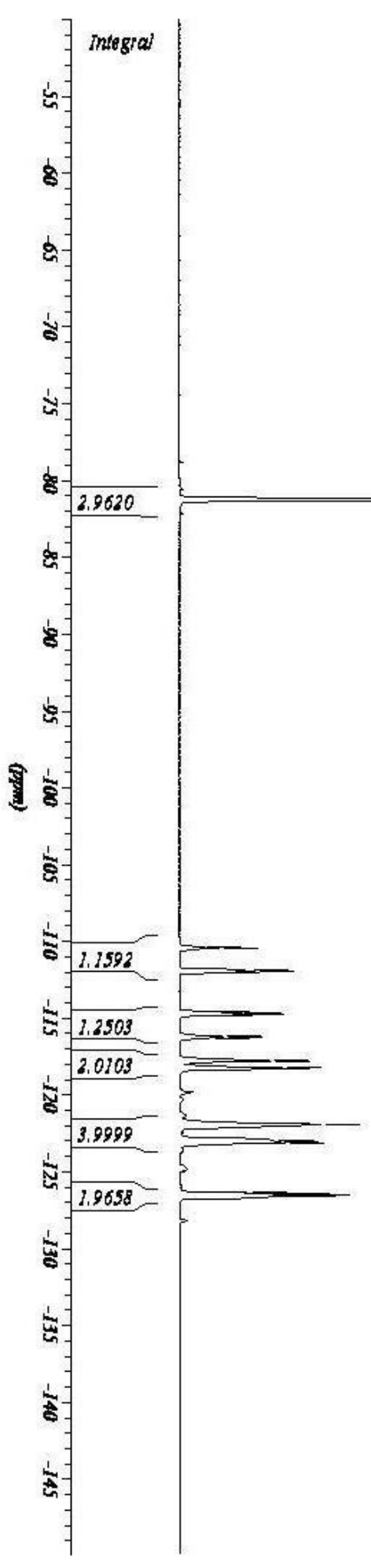

$\Gamma_{-81.2422}^{-81.2895}$
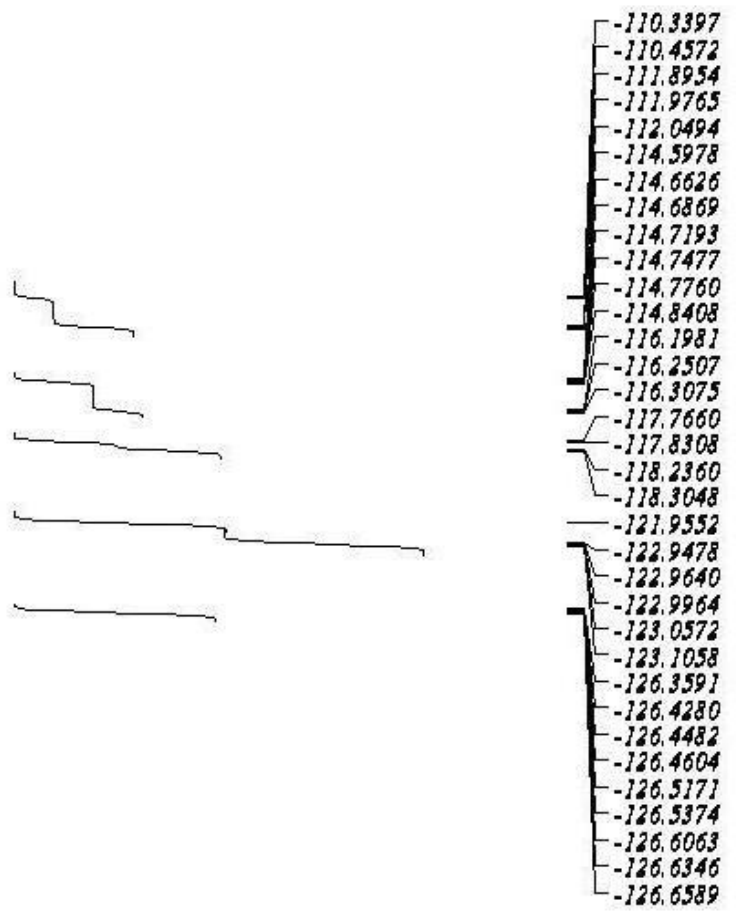


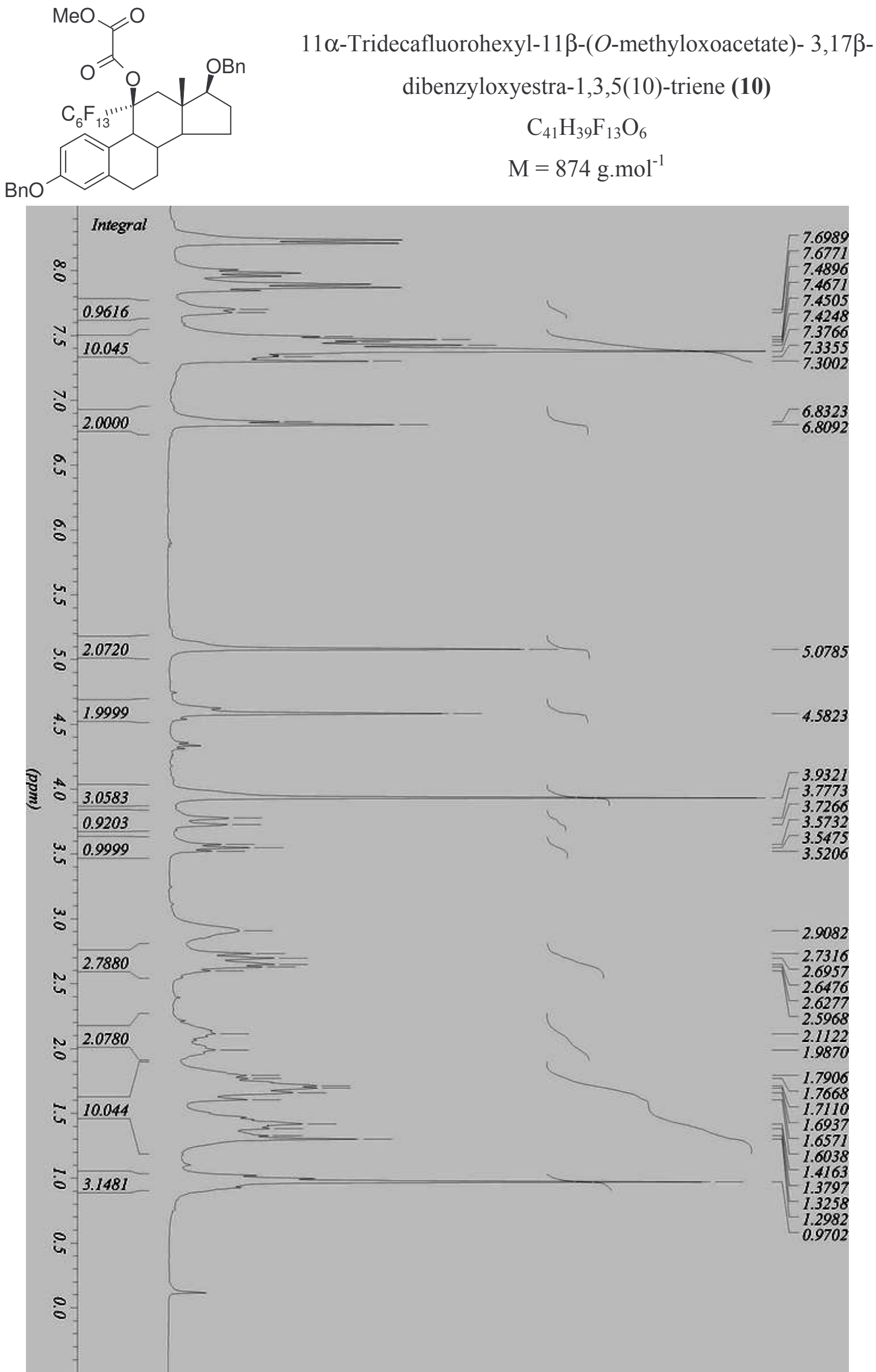




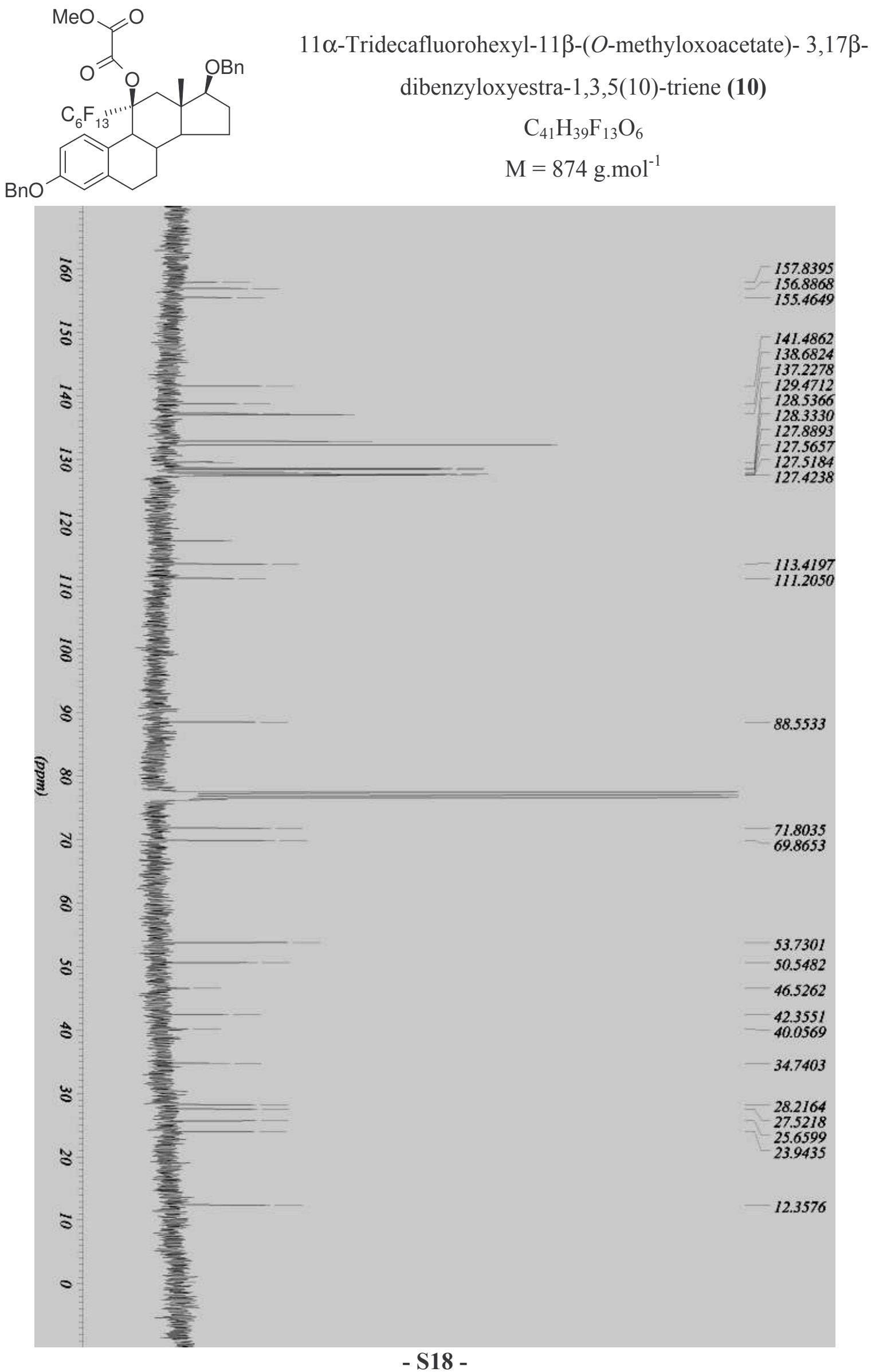




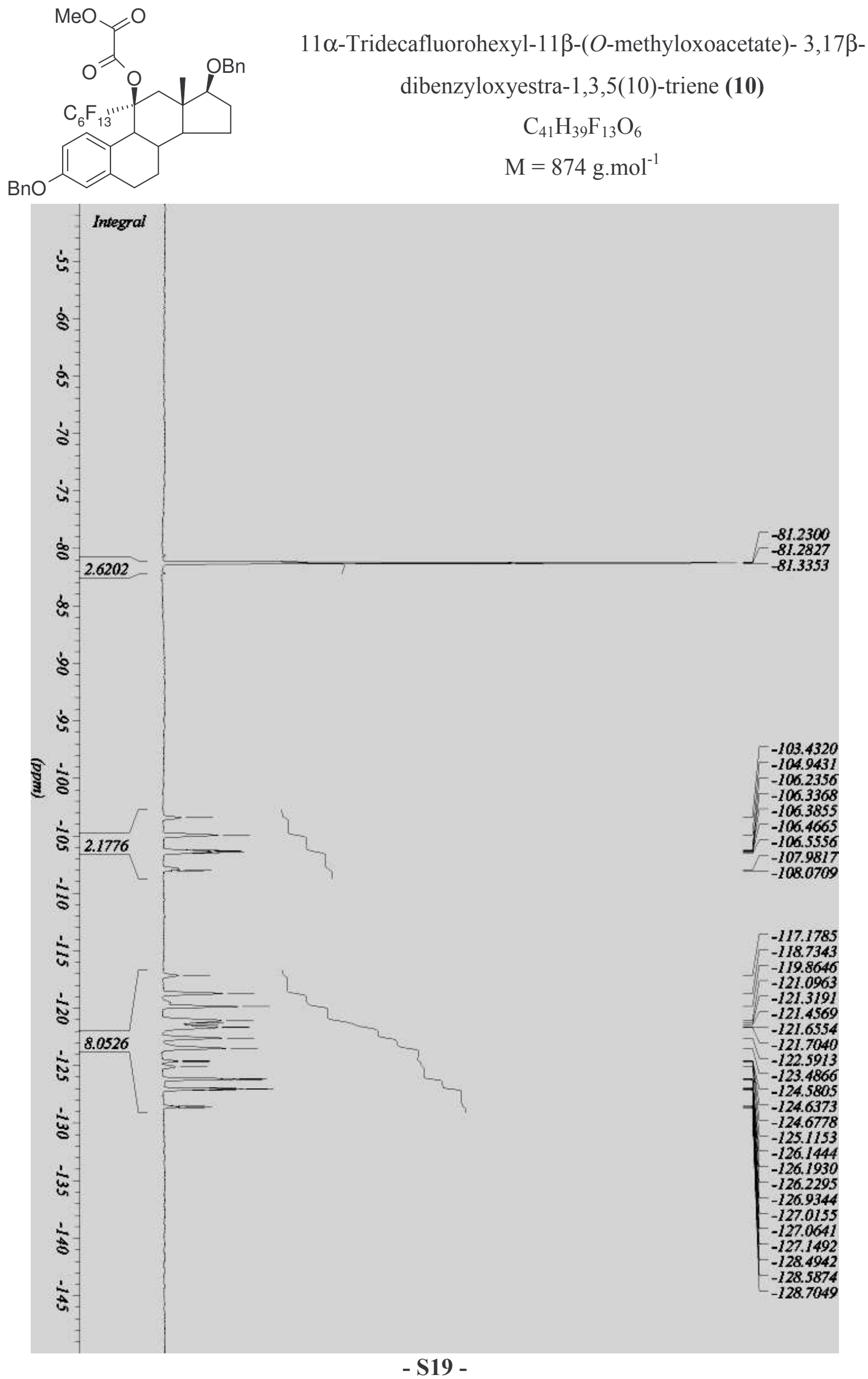




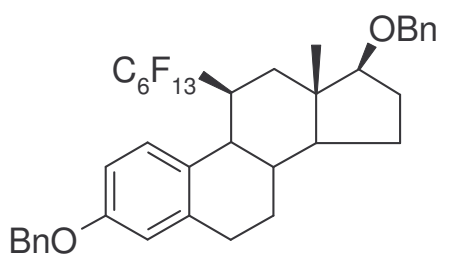

11ß-Tridecafluorohexyl-3,17 $\beta$-dibenzyloxyestra-1,3,5(10)-

$$
\begin{gathered}
\text { triene (11a) } \\
\mathrm{C}_{38} \mathrm{H}_{37} \mathrm{~F}_{13} \mathrm{O}_{2} \\
\mathrm{M}=770 \text { g.mol }{ }^{-1}
\end{gathered}
$$

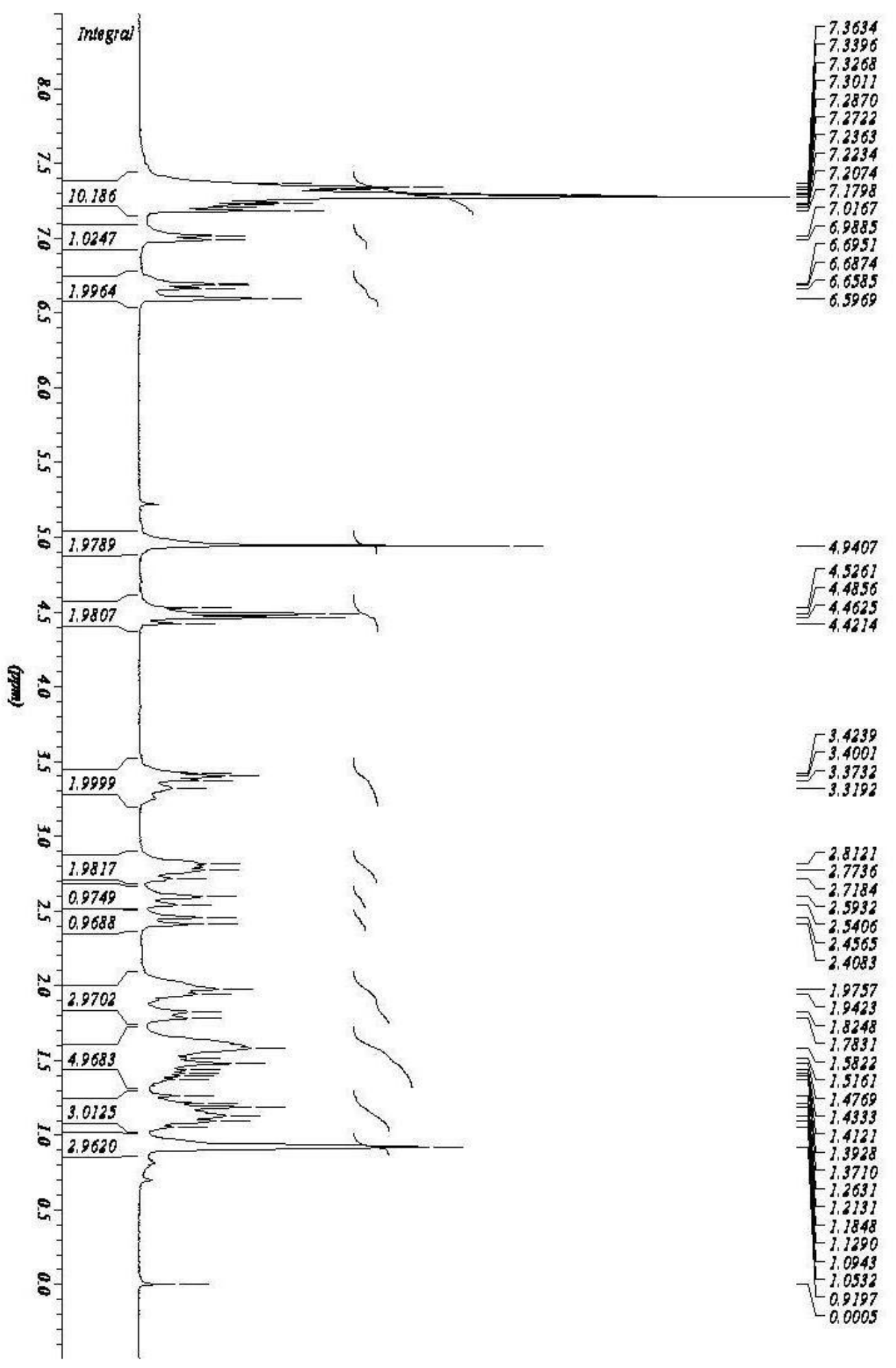


<smiles>C[C@H](Cc1ccccc1)C[C@@]1(C)C2CC[C@@H](OCc3ccccc3)C2C2CCc3cc(OCc4ccccc4)ccc3C21</smiles>

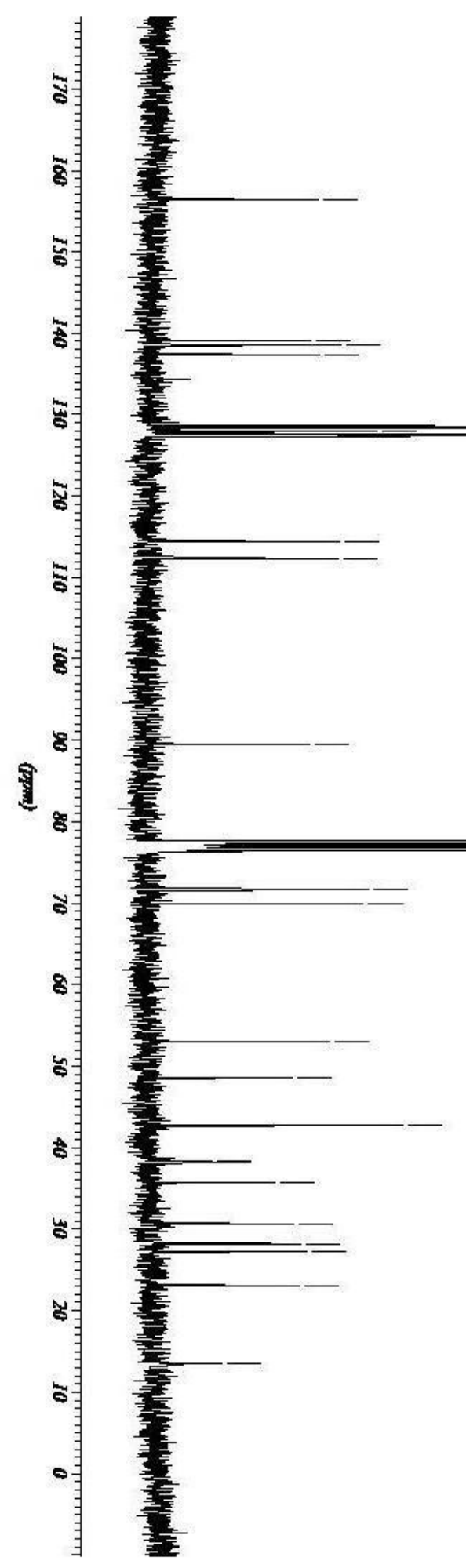

$11 \beta$-Tridecafluorohexyl-3,17 $\beta$-dibenzyloxyestra-1,3,5(10)-

$$
\begin{gathered}
\text { triene (11a) } \\
\mathrm{C}_{38} \mathrm{H}_{37} \mathrm{~F}_{13} \mathrm{O}_{2} \\
\mathrm{M}=770 \text { g.mol }{ }^{-1}
\end{gathered}
$$

$-156.4177$

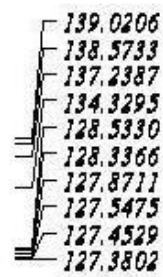

$-114,4015$ 112.3287

$-89,4951$

$-53.0428$

-48.508 ?

$-42.6897$

$-38.3477$

$-38,2022$

38.1004

35,6531

$-30.6092$

38.2382

27.2164

$-13,4740$ 
<smiles>C[C@H](C1c2ccc(OCc3ccccc3)cc2CCC1C1CC[C@@H](O)[C@@]1(C)Cc1ccccc1)C(F)(F)F</smiles>

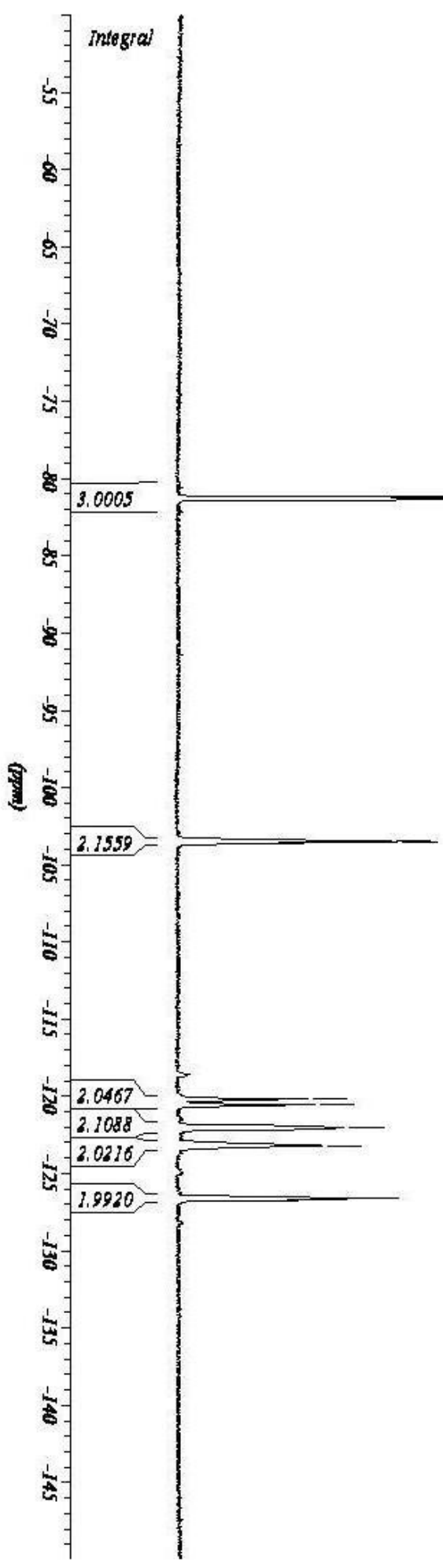

$11 \beta$-Tridecafluorohexyl-3,17 $\beta$-dibenzyloxyestra-1,3,5(10)triene (11a)

$$
\mathrm{C}_{38} \mathrm{H}_{37} \mathrm{~F}_{13} \mathrm{O}_{2}
$$$$
\mathrm{M}=770 \mathrm{~g} \cdot \mathrm{mol}^{-1}
$$ 


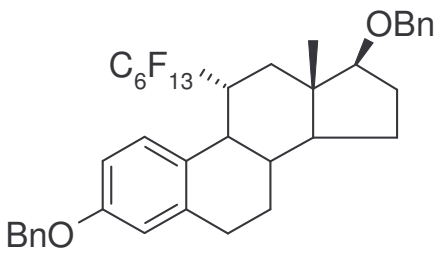

$11 \alpha$-Tridecafluorohexyl-3,17 $\beta$-dibenzyloxyestra-1,3,5(10)-

$$
\begin{gathered}
\text { triene (11b) } \\
\mathrm{C}_{38} \mathrm{H}_{37} \mathrm{~F}_{13} \mathrm{O}_{2} \\
\mathrm{M}=770 \text { g.mol }{ }^{-1}
\end{gathered}
$$

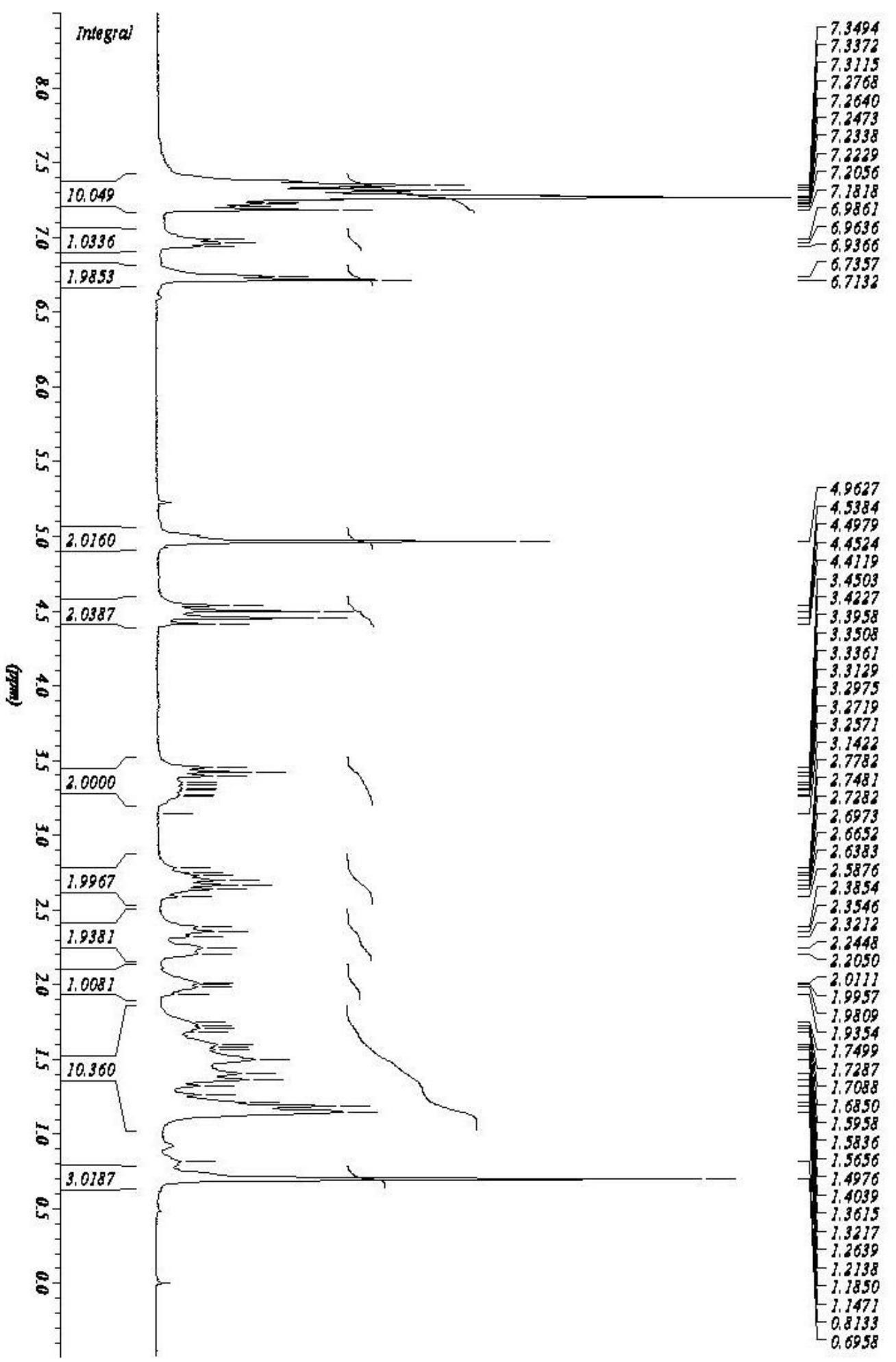


<smiles>CC1(C(=O)c2ccccc2)CCC2C3CCc4cc(OCc5ccccc5)ccc4C3C2C1</smiles>

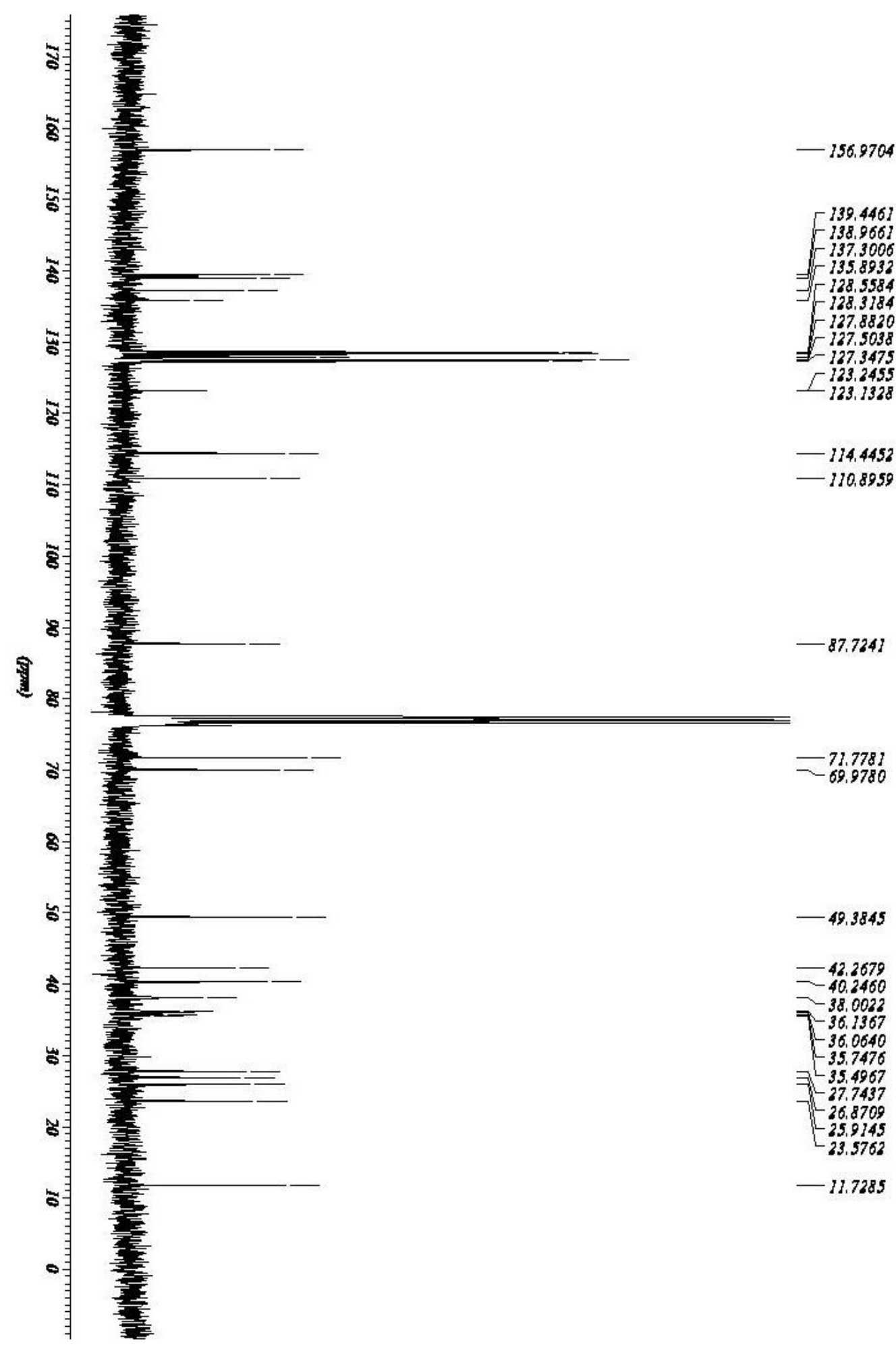

triene (11b)

$\mathrm{C}_{38} \mathrm{H}_{37} \mathrm{~F}_{13} \mathrm{O}_{2}$

$\mathrm{M}=770$ g. $\mathrm{mol}^{-1}$ 
<smiles>CCc1cc(OCc2ccccc2)ccc1C1C2CCC(Cc3ccccc3)C2(C)CC[C@@H]1C(C)(C)F</smiles>

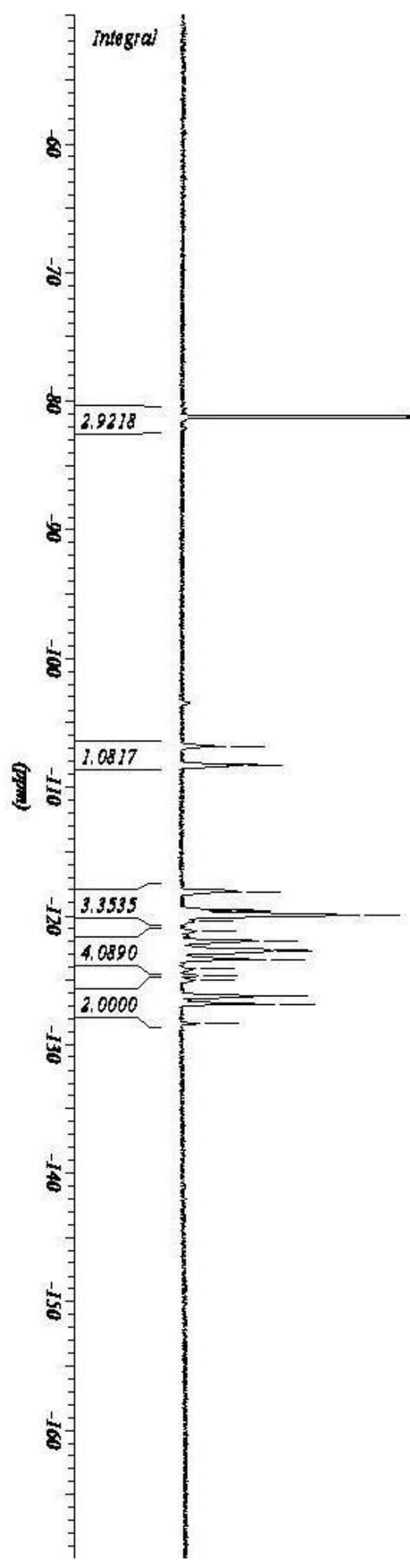

$11 \alpha$-Tridecafluorohexyl-3,17 $\beta$-dibenzyloxyestra-1,3,5(10)triene (11b)

$$
\mathrm{C}_{38} \mathrm{H}_{37} \mathrm{~F}_{13} \mathrm{O}_{2}
$$$$
\mathrm{M}=770 \mathrm{~g} \cdot \mathrm{mol}^{-1}
$$ 
<smiles></smiles>

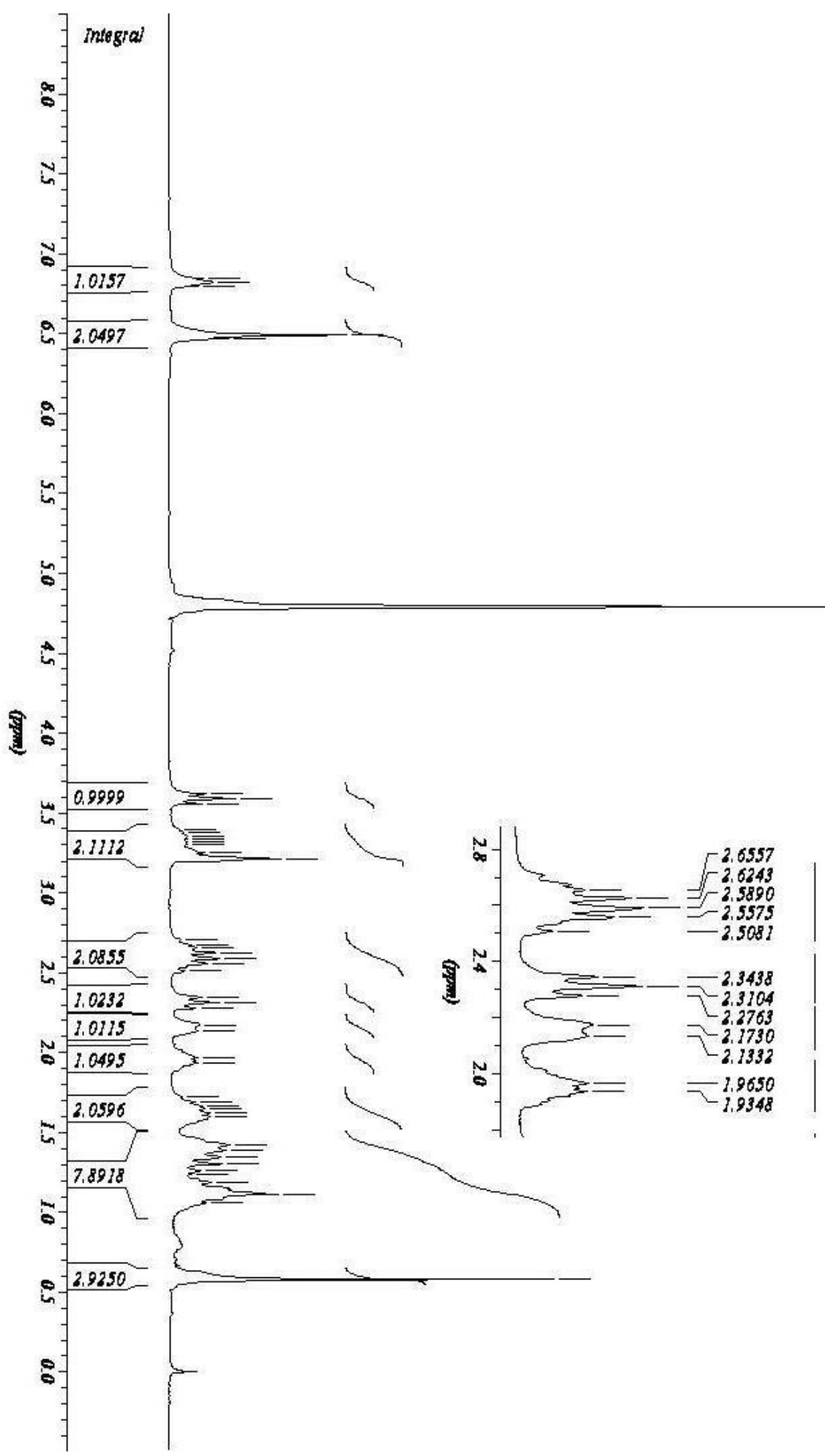

$11 \alpha$-Tridecafluorohexyl-3,17 $\beta$-dibenzyloxyestra-1,3,5(10)triene (12)

$$
\mathrm{C}_{24} \mathrm{H}_{23} \mathrm{~F}_{13} \mathrm{O}_{2}
$$$$
\mathrm{M}=590 \mathrm{~g} \cdot \mathrm{mol}^{-1}
$$

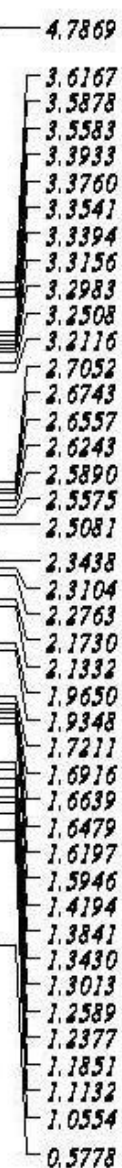


<smiles>CC1(C(F)(F)F)C[C@@H](O)C2c3ccc(O)cc3CCC2C2CCC(O)C21</smiles>

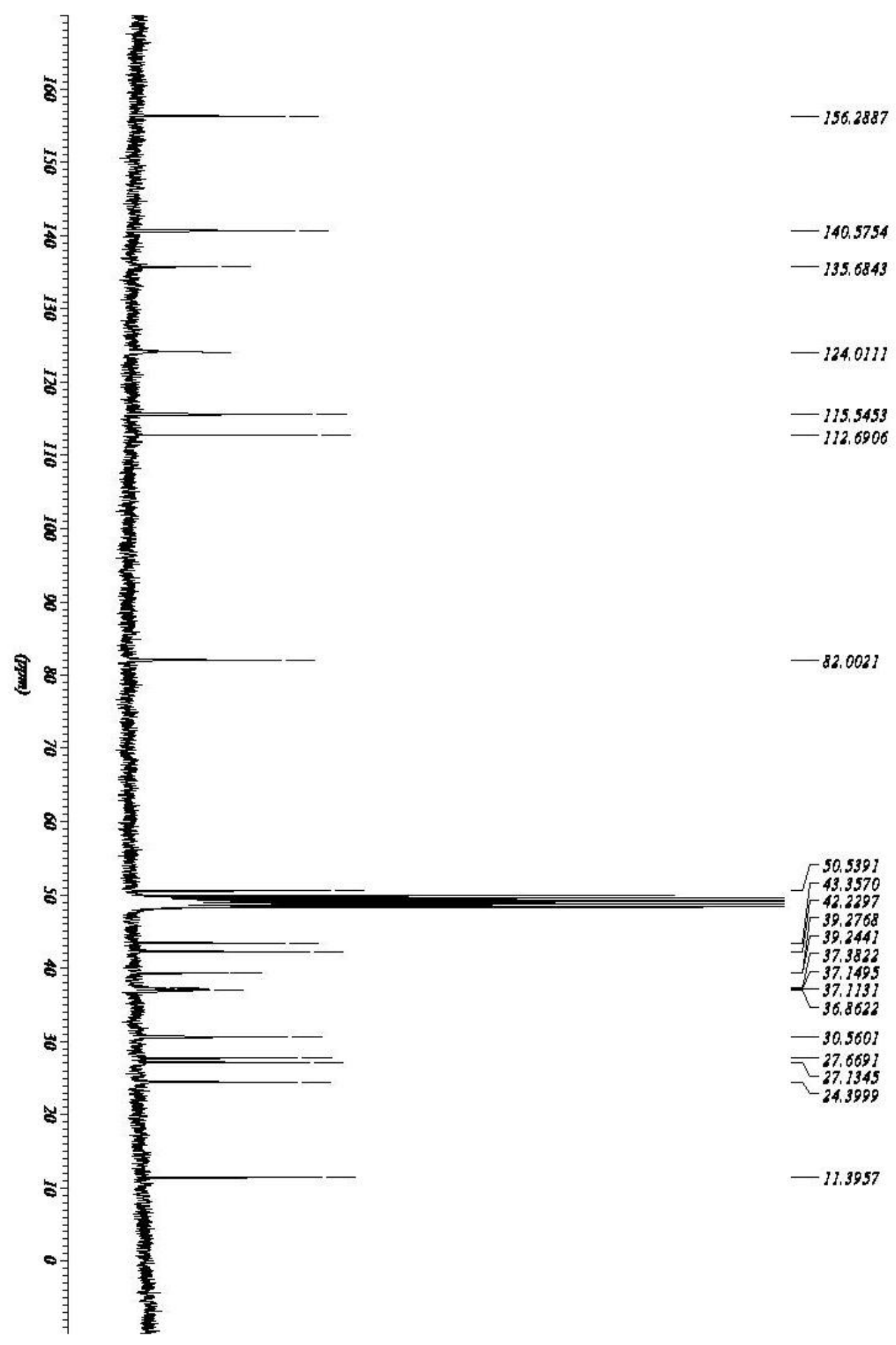

$11 \alpha$-Tridecafluorohexyl-3,17 $\beta$-dibenzyloxyestra-1,3,5(10)-

$$
\begin{gathered}
\text { triene (12) } \\
\mathrm{C}_{24} \mathrm{H}_{23} \mathrm{~F}_{13} \mathrm{O}_{2} \\
\mathrm{M}=590 \mathrm{~g} \cdot \mathrm{mol}^{-1}
\end{gathered}
$$


<smiles></smiles>

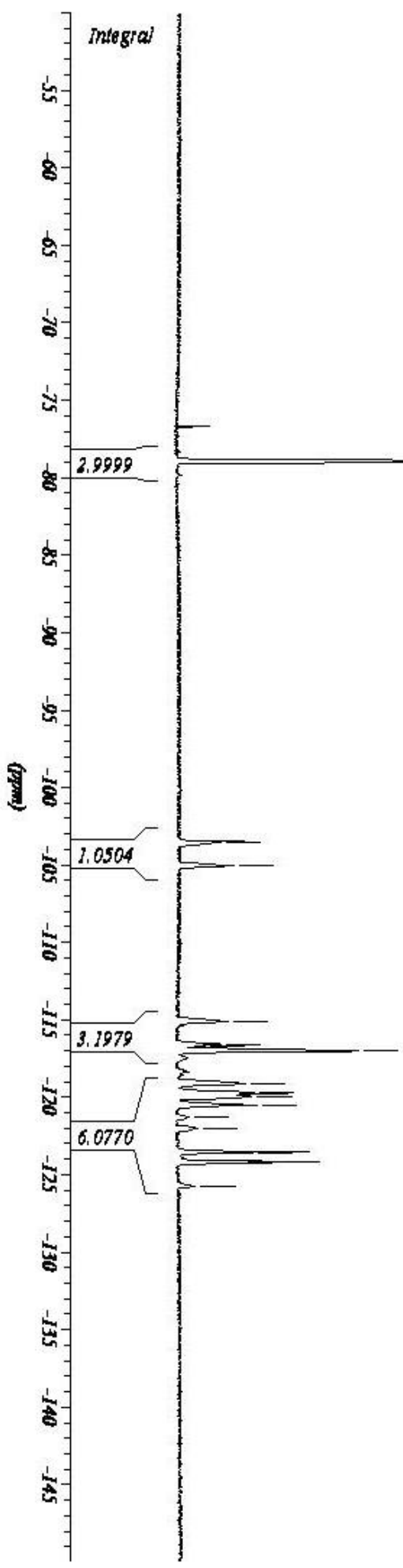

$11 \alpha$-Tridecafluorohexyl-3,17 $\beta$-dibenzyloxyestra-1,3,5(10)triene (12)

$\mathrm{C}_{24} \mathrm{H}_{23} \mathrm{~F}_{13} \mathrm{O}_{2}$

$\mathrm{M}=590 \mathrm{~g} \cdot \mathrm{mol}^{-1}$
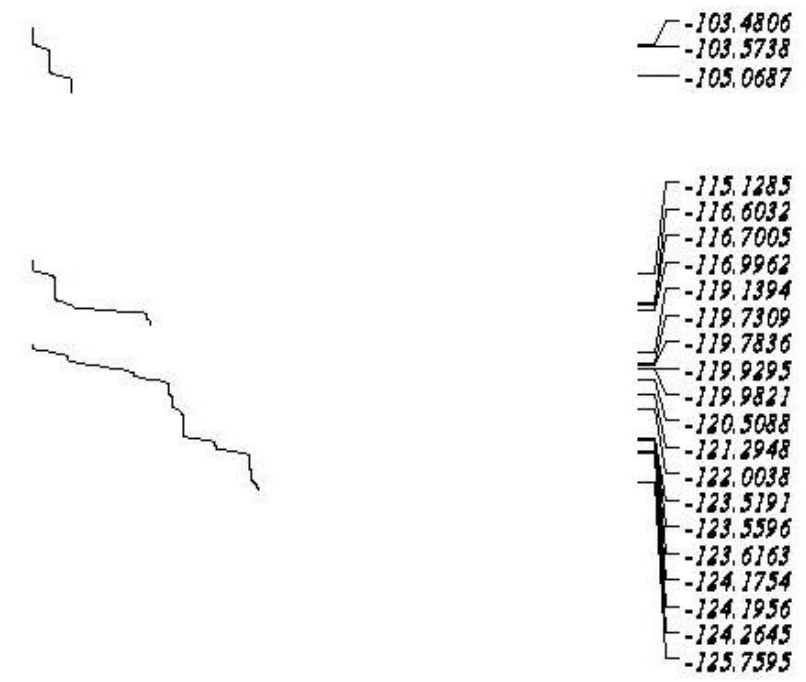


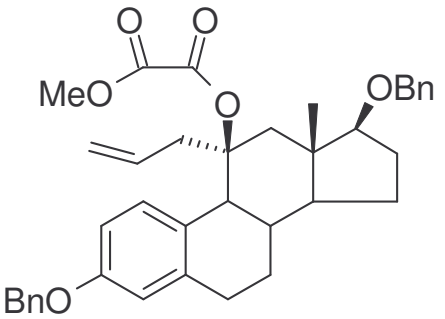

$11 \alpha$-Allyl-11 $\beta$-(O-methyloxoacetate)-3,17 $\beta$ dibenzyloxyestra-1,3,5(10)-triene (14a)

$$
\begin{gathered}
\mathrm{C}_{38} \mathrm{H}_{42} \mathrm{O}_{6} \\
\mathrm{M}=594 \mathrm{~g} \cdot \mathrm{mol}^{-1}
\end{gathered}
$$

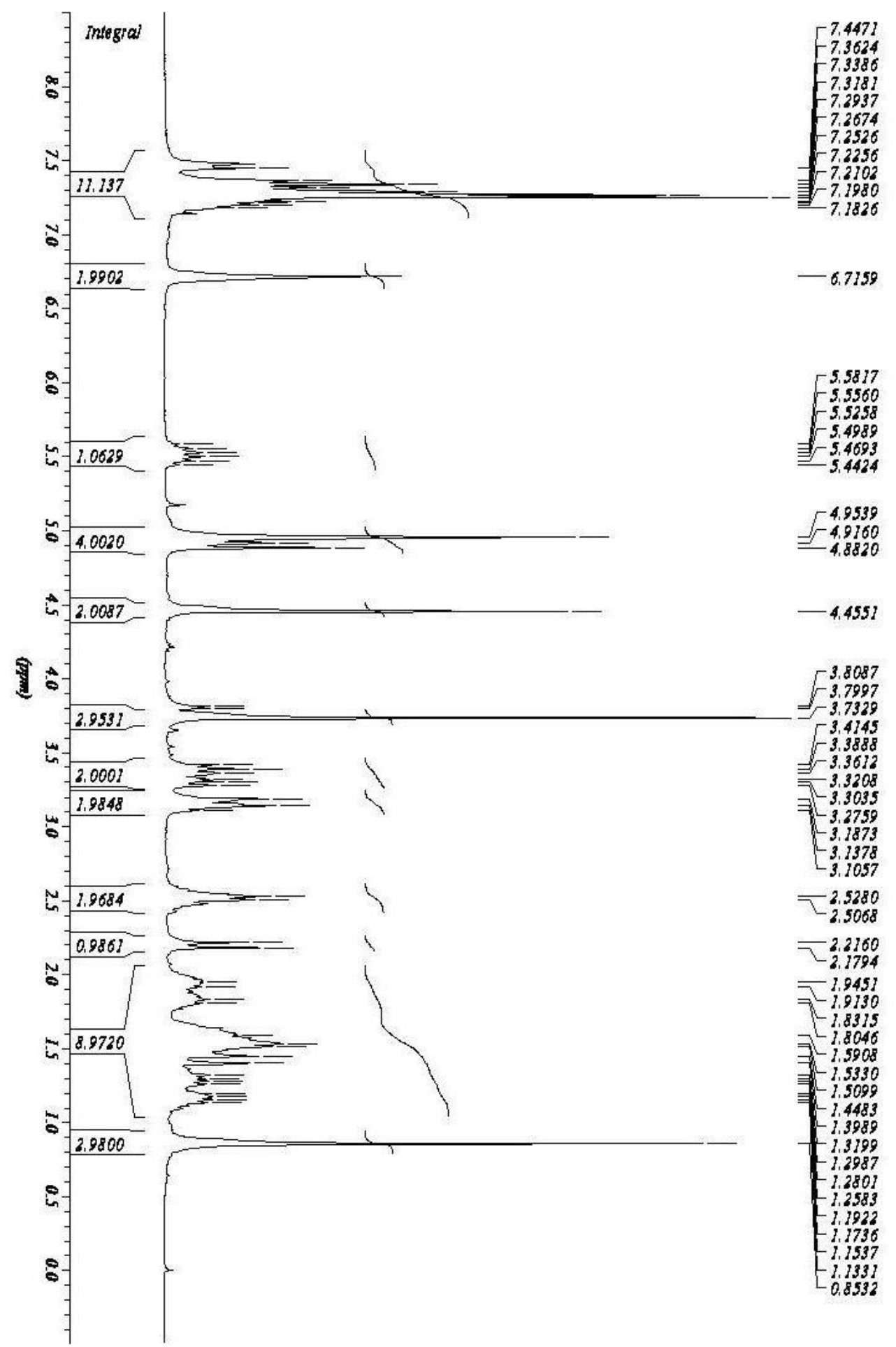


<smiles>C=CC[C@]1(OC(=O)C(=O)OC)C[C@]2(C)C(Cc3ccccc3)CCC2C2CCc3cc(OCc4ccccc4)ccc3C21</smiles>

$11 \alpha$-Allyl-11 $\beta$-( $O$-methyloxoacetate)-3,17 $\beta$ dibenzyloxyestra-1,3,5(10)-triene (14a)

$$
\begin{gathered}
\mathrm{C}_{38} \mathrm{H}_{42} \mathrm{O}_{6} \\
\mathrm{M}=594 \mathrm{~g} \cdot \mathrm{mol}^{-1}
\end{gathered}
$$

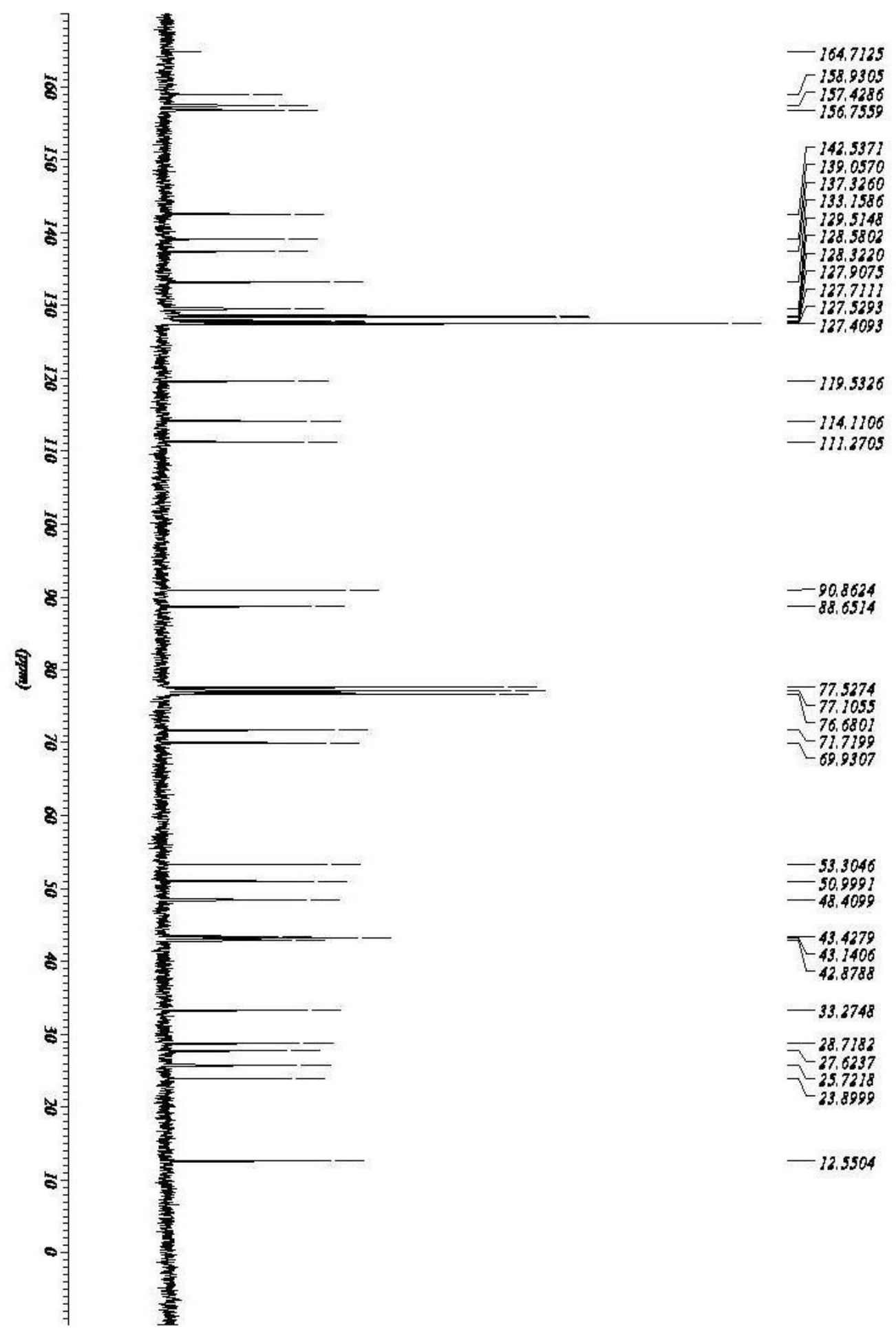


<smiles>C=CC[C@@H]1CC2(C)C(OCc3ccccc3)CCC2C2CCc3cc(OCc4ccccc4)ccc3C21</smiles>

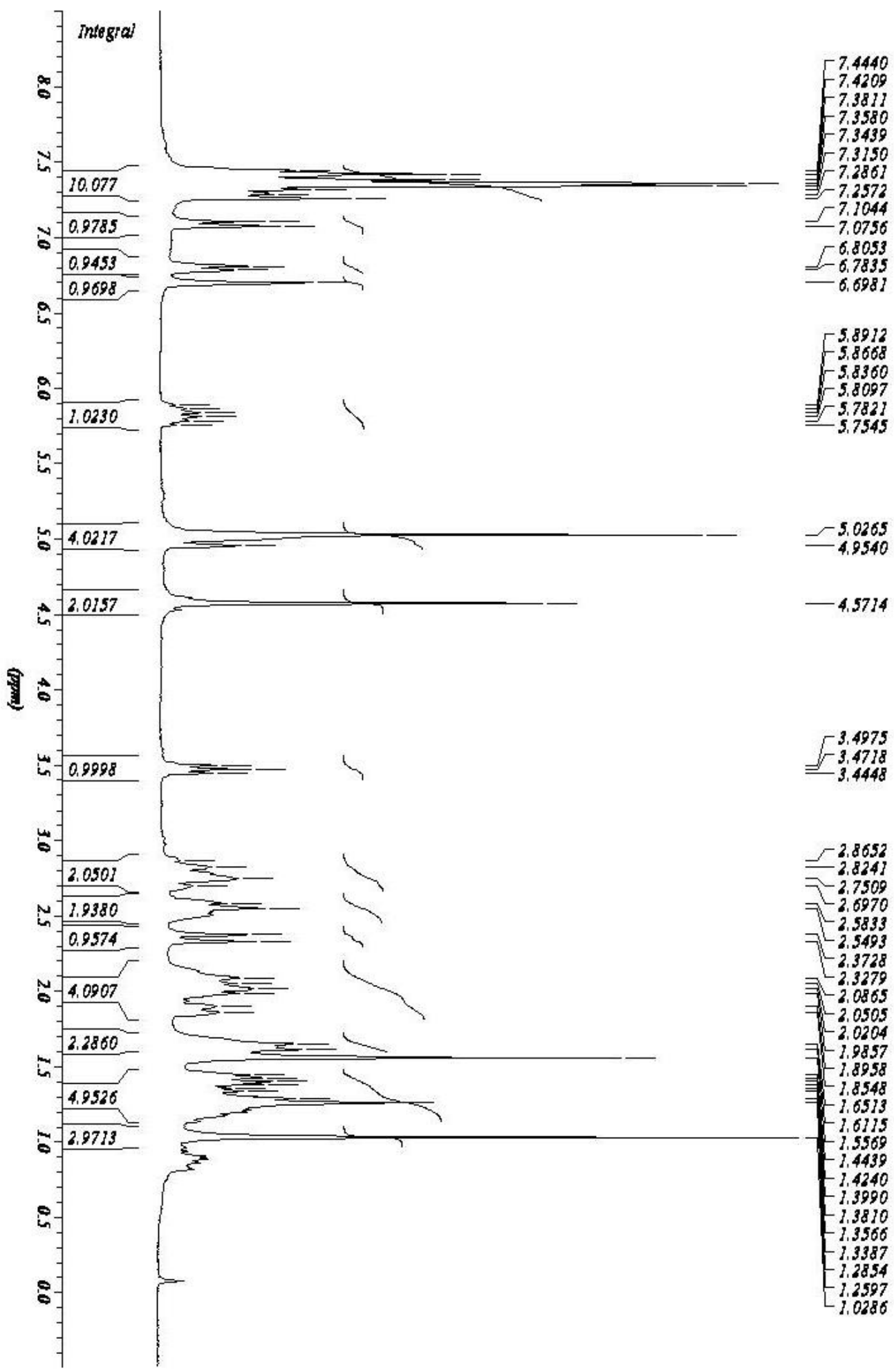




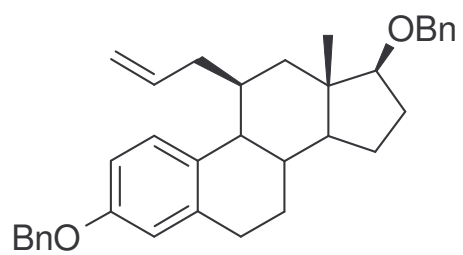

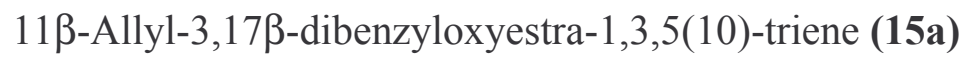

$$
\begin{gathered}
\mathrm{C}_{35} \mathrm{H}_{40} \mathrm{O}_{2} \\
\mathrm{M}=492 \mathrm{~g} \cdot \mathrm{mol}^{-1}
\end{gathered}
$$

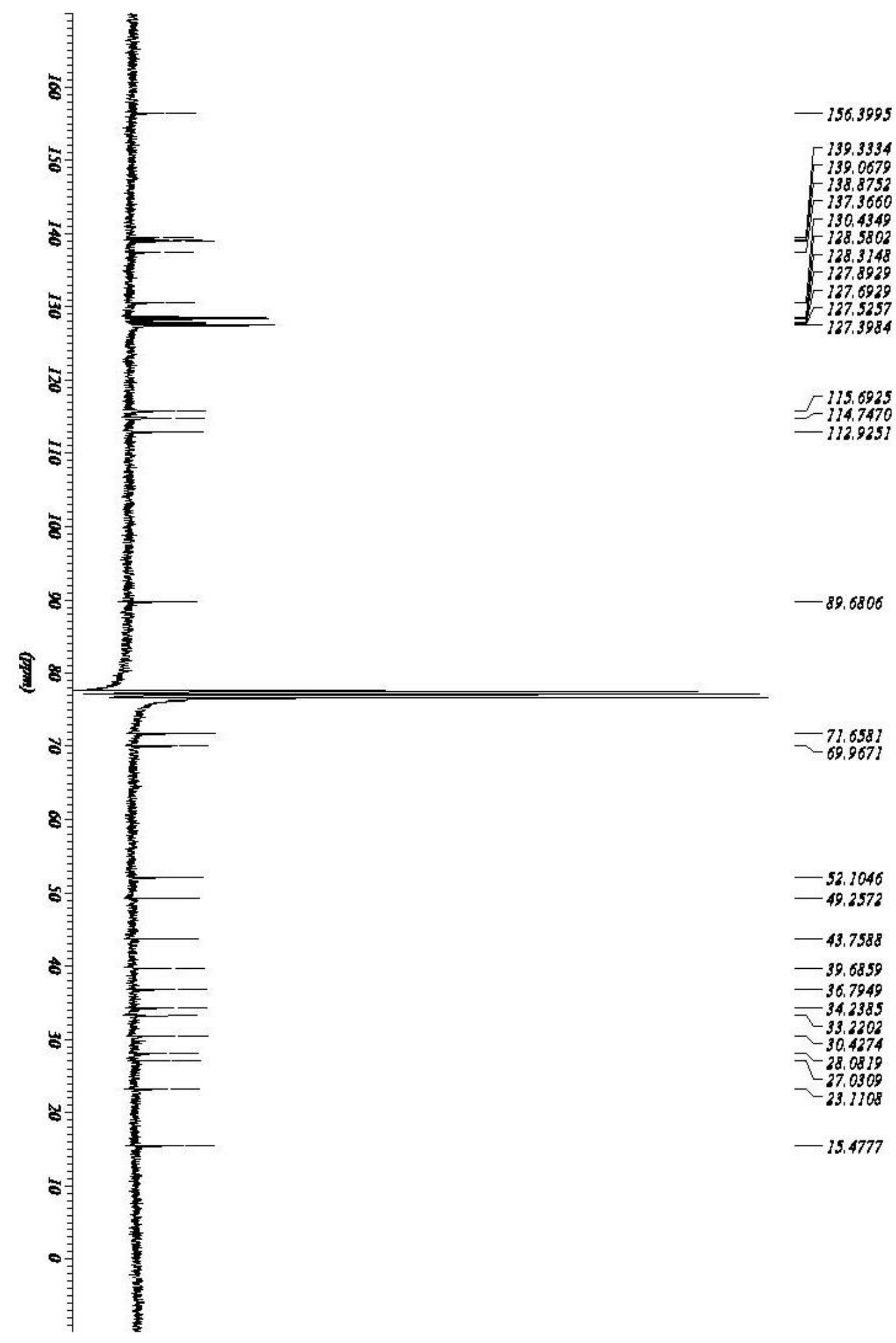


<smiles>C[C@]12C[C@H](c3ccccc3)C3c4ccc(OCc5ccccc5)cc4CCC3C1CC[C@@H]2OCc1ccccc1</smiles>

$11 \alpha$-Phenyl-3,17 $\beta$-dibenzyloxyestra-1,3,5(10)-triene (15b)

$$
\begin{gathered}
\mathrm{C}_{38} \mathrm{H}_{40} \mathrm{O}_{2} \\
\mathrm{M}=528 \mathrm{~g} \cdot \mathrm{mol}^{-1}
\end{gathered}
$$

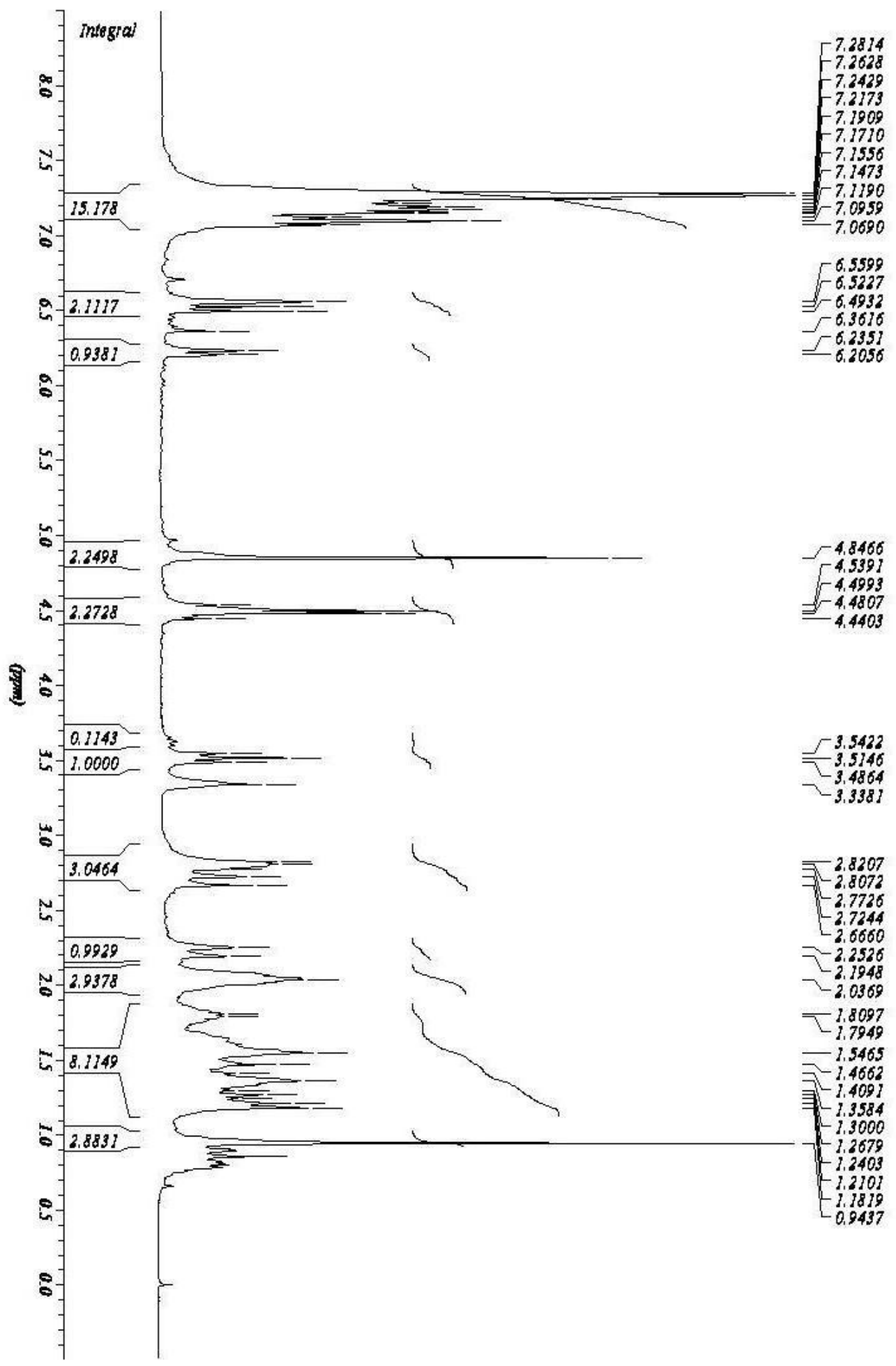


<smiles>CC(Cc1ccccc1)OC1CCC2C3CCc4cc(OCc5ccccc5)ccc4C3[C@H](c3ccccc3)C[C@]12C</smiles>

11 $\alpha$-Phenyl-3,17 $\beta$-dibenzyloxyestra-1,3,5(10)-triene (15b)

$$
\begin{gathered}
\mathrm{C}_{38} \mathrm{H}_{40} \mathrm{O}_{2} \\
\mathrm{M}=528 \mathrm{~g} \cdot \mathrm{mol}^{-1}
\end{gathered}
$$

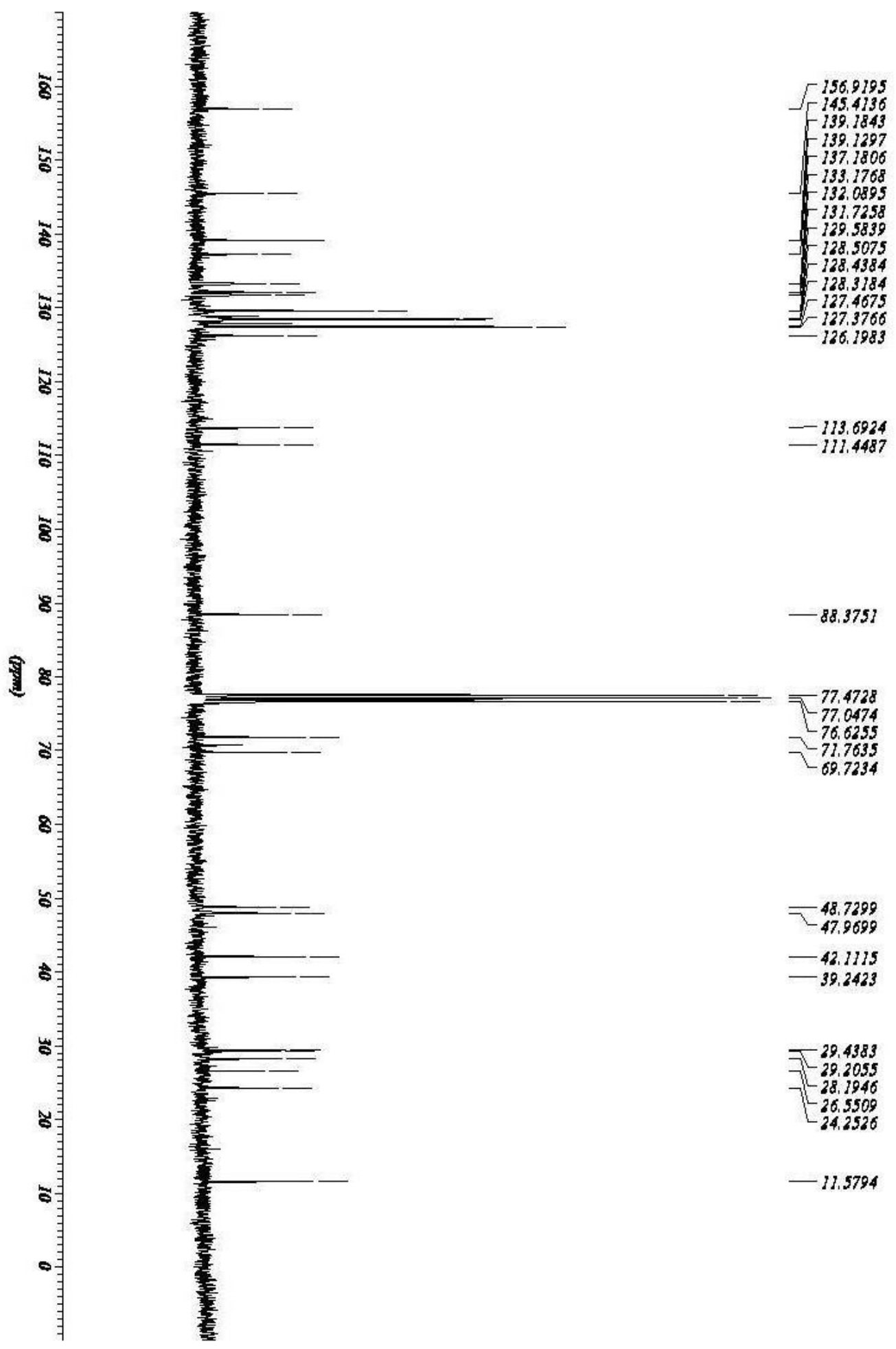




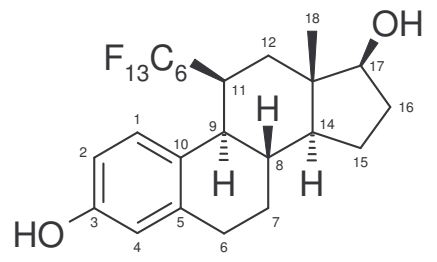

$11 \beta$-Tridecafluorohexyl-3,17 $\beta$-dibenzyloxyestra-1,3,5(10)-

$$
\begin{gathered}
\text { triene (1e) } \\
\mathrm{C}_{38} \mathrm{H}_{37} \mathrm{~F}_{13} \mathrm{O}_{2} \\
\mathrm{M}=770 \mathrm{~g} \cdot \mathrm{mol}^{-1}
\end{gathered}
$$

\begin{tabular}{|c|c|c|c|}
\hline Position identifier & $\delta^{13} \mathrm{C}(\mathrm{ppm})$ & $\delta^{1} H(p p m)$ & Multiplicity \\
\hline 1 & 130.1 & 6.95 & $\mathrm{~d}(J=8.7 \mathrm{~Hz})$ \\
\hline 2 & 115.9 & 6.43 & $\begin{array}{c}\operatorname{dd}(J=8.7 H z, J=2.7 \\
H z)\end{array}$ \\
\hline 3 & 155.7 & - & - \\
\hline 4 & 113.7 & 6.36 & - \\
\hline 5 & 128.3 or 139.7 & - & - \\
\hline 6 & 31.7 & $2.45-2.69$ & $\mathrm{~m}$ \\
\hline 7 & 28.5 & steroid envelope & - \\
\hline 8 & 37.6 & steroid envelope & - \\
\hline 9 & 49.7 & 2.81 & $\begin{array}{c}\mathrm{dd}(J=10.5 \mathrm{~Hz}, J= \\
4.5 \mathrm{~Hz})\end{array}$ \\
\hline 10 & 128.3 or 139.7 & - & - \\
\hline 11 & 39.6 & 3.42 & broad t $\left(J_{H F}=21 \mathrm{~Hz}\right)$ \\
\hline 12 & 38.4 & 2.30 & $\mathrm{t}(J=7.3 \mathrm{~Hz})$ \\
\hline 13 & 43.7 & - & - \\
\hline 14 & 53.8 & steroid envelope & - \\
\hline 15 & 24.0 & steroid envelope & - \\
\hline 16 & 31.0 & steroid envelope & - \\
\hline 17 & 83.8 & 3.58 & $\mathrm{t}(J=7.3 \mathrm{~Hz})$ \\
\hline 18 & $13.5(\mathrm{t})$ & 0.78 & broad s \\
\hline
\end{tabular}

Key of the $\beta$-stereochemistry at center 11 is supposedly the through space coupling observed for $\mathrm{C}_{18}$. Besides, the pattern for $\mathrm{H}_{9}$ at $2.81 \mathrm{ppm}$ is pointing for a cis relationship with both $\mathrm{H}_{8}$ and $\mathrm{H}_{11}$ (see below).
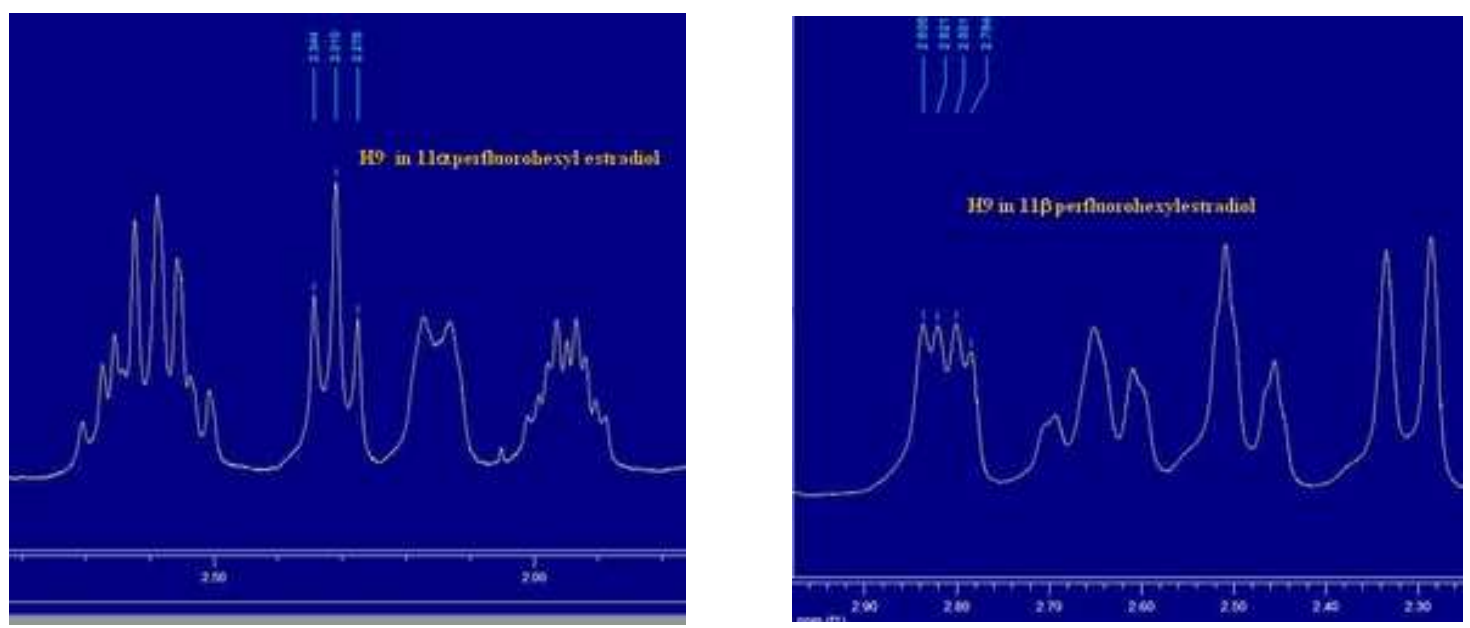
<smiles>C[C@@H]1C[C@@]2(N)[C@@H](CC[C@H]2O)[C@H]2CCc3cc(O)ccc3[C@@H]21</smiles>

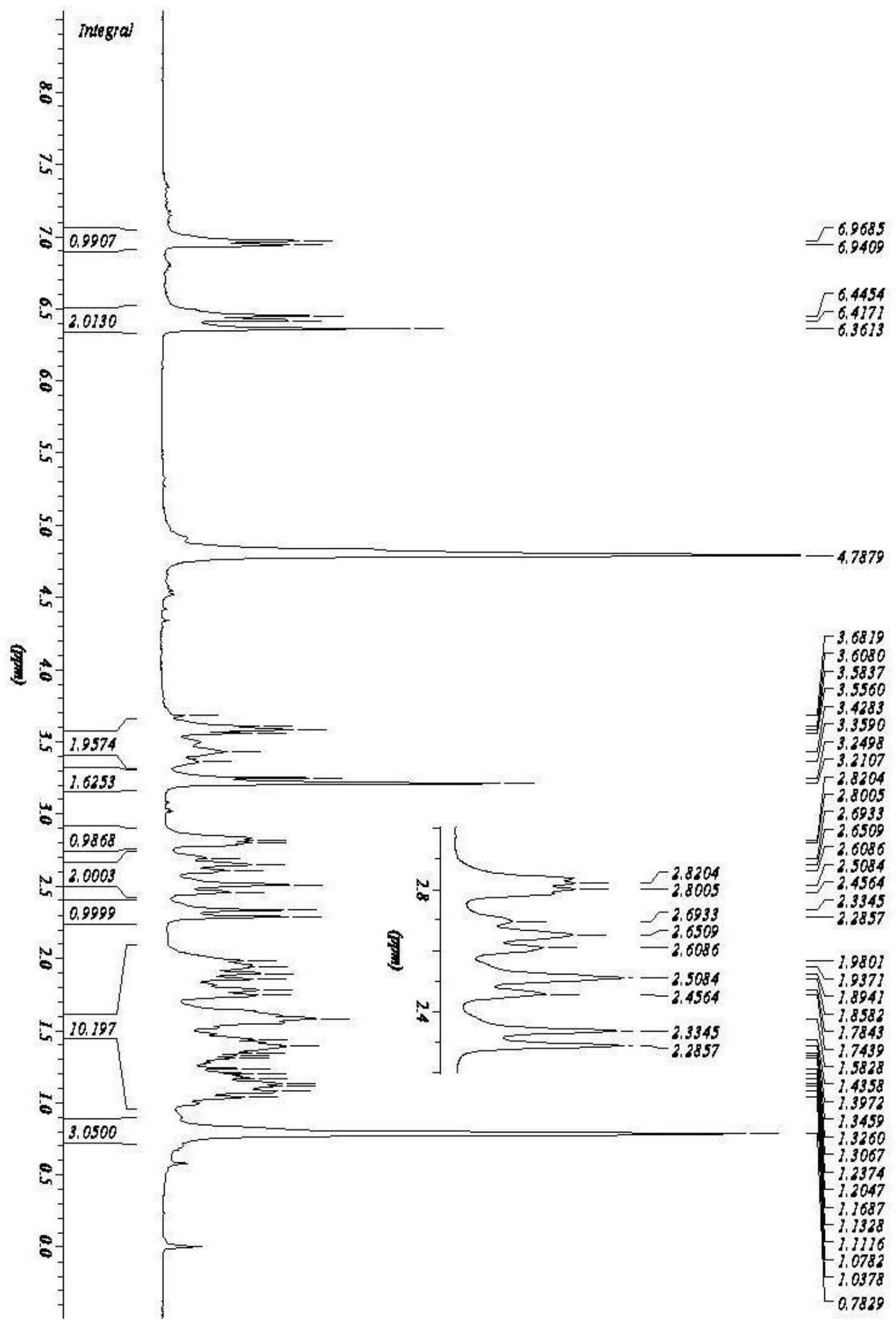

triene (1e)

$\mathrm{C}_{38} \mathrm{H}_{37} \mathrm{~F}_{13} \mathrm{O}_{2}$

$\mathrm{M}=770 \mathrm{~g} \cdot \mathrm{mol}^{-1}$ 

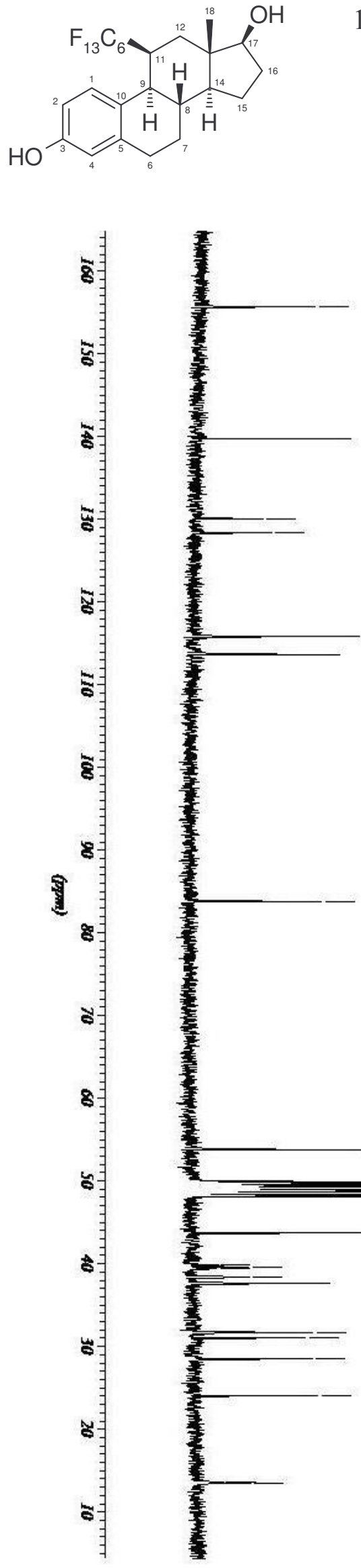

$11 \beta$-Tridecafluorohexyl-3,17 $\beta$-dibenzyloxyestra-1,3,5(10)triene (1e)

$$
\mathrm{C}_{38} \mathrm{H}_{37} \mathrm{~F}_{13} \mathrm{O}_{2}
$$$$
\mathrm{M}=770 \mathrm{~g} \cdot \mathrm{mol}^{-1}
$$

$-155.6851$

$-139.7288$

$\begin{array}{r}-130.0550 \\ -128.3094 \\ \hline\end{array}$

115.8435
-113.6652

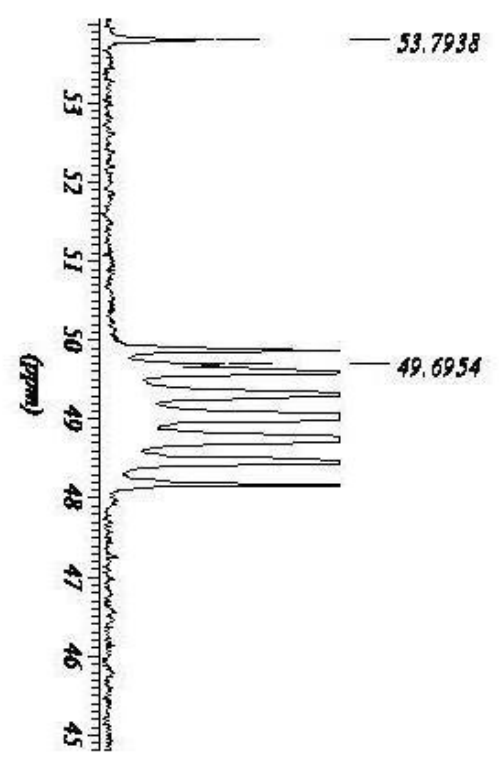

$-83.7622$

$-53.7938$

$-49.695$

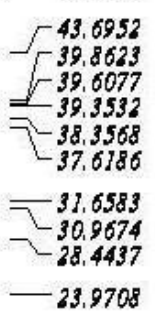

$\left[\begin{array}{l}13.5122 \\ -13.4576 \\ 13.3631\end{array}\right.$ 


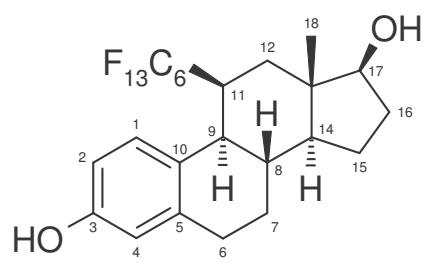
$11 \beta$-Tridecafluorohexyl-3,17 $\beta$-dibenzyloxyestra-1,3,5(10)- triene (1e)
$\mathrm{C}_{38} \mathrm{H}_{37} \mathrm{~F}_{13} \mathrm{O}_{2}$
$\mathrm{M}=770 \mathrm{~g} \cdot \mathrm{mol}^{-1}$

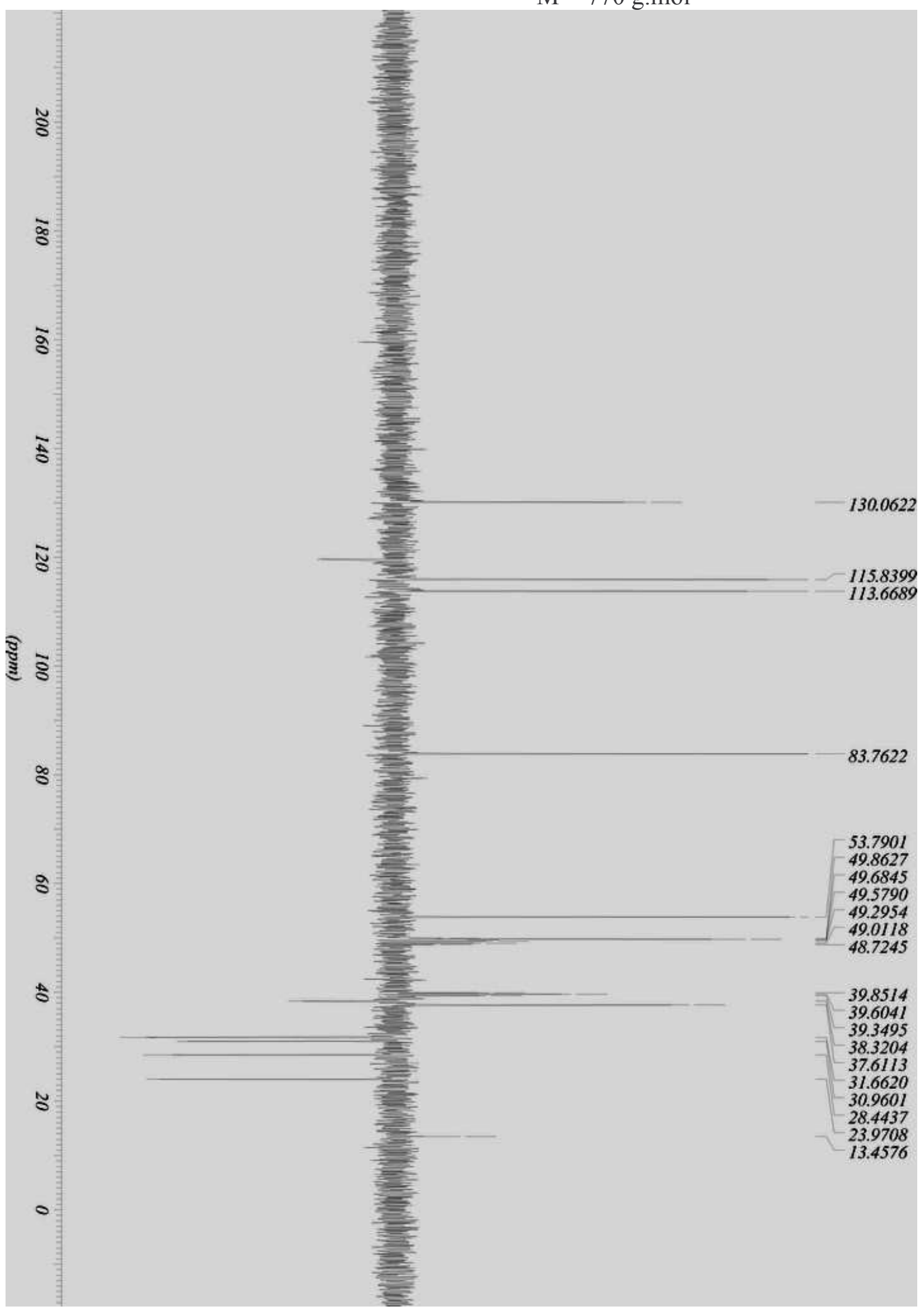


<smiles>C[C@@H]1C[C@]2(C)[C@@H](O)CC[C@H]2[C@H]2CCc3cc(O)ccc3[C@H]12</smiles>

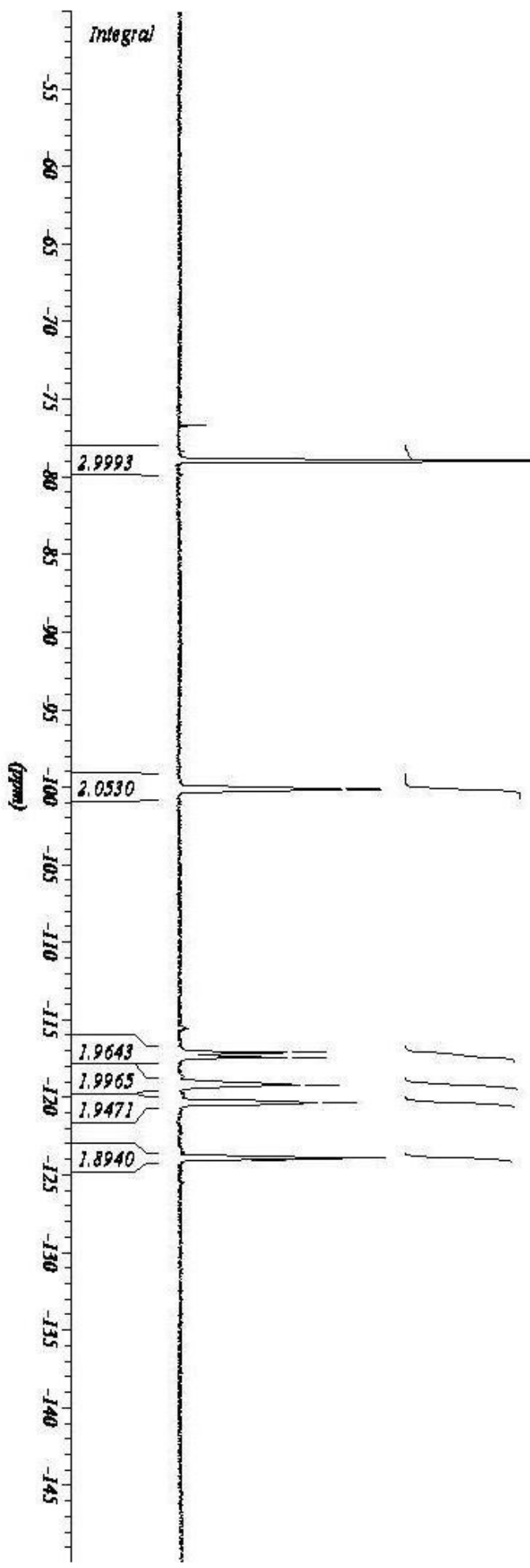

$11 \beta$-Tridecafluorohexyl-3,17ß-dibenzyloxyestra-1,3,5(10)triene (1e)

$$
\mathrm{C}_{38} \mathrm{H}_{37} \mathrm{~F}_{13} \mathrm{O}_{2}
$$$$
\mathrm{M}=770 \mathrm{~g} \cdot \mathrm{mol}^{-1}
$$ 

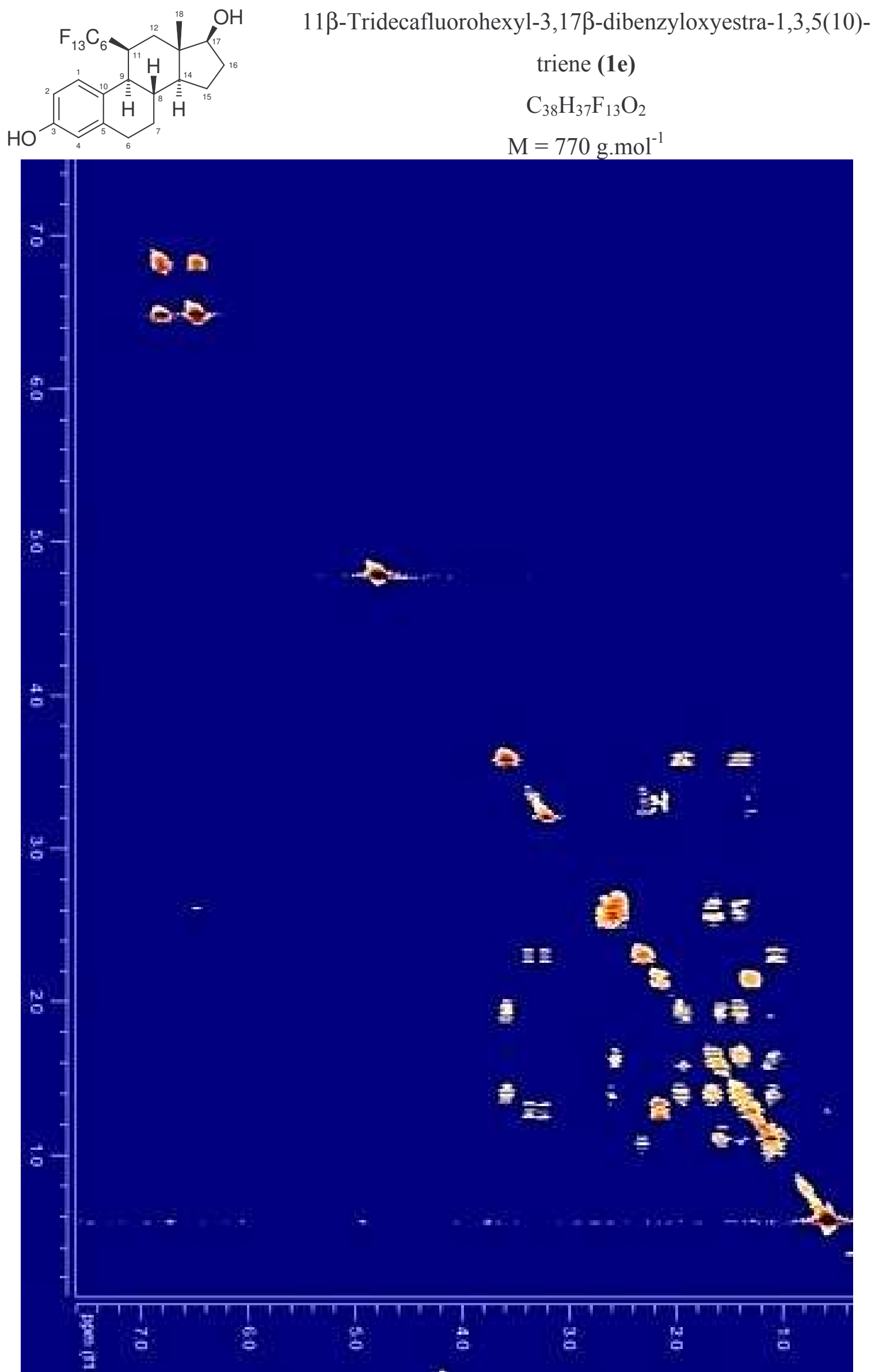

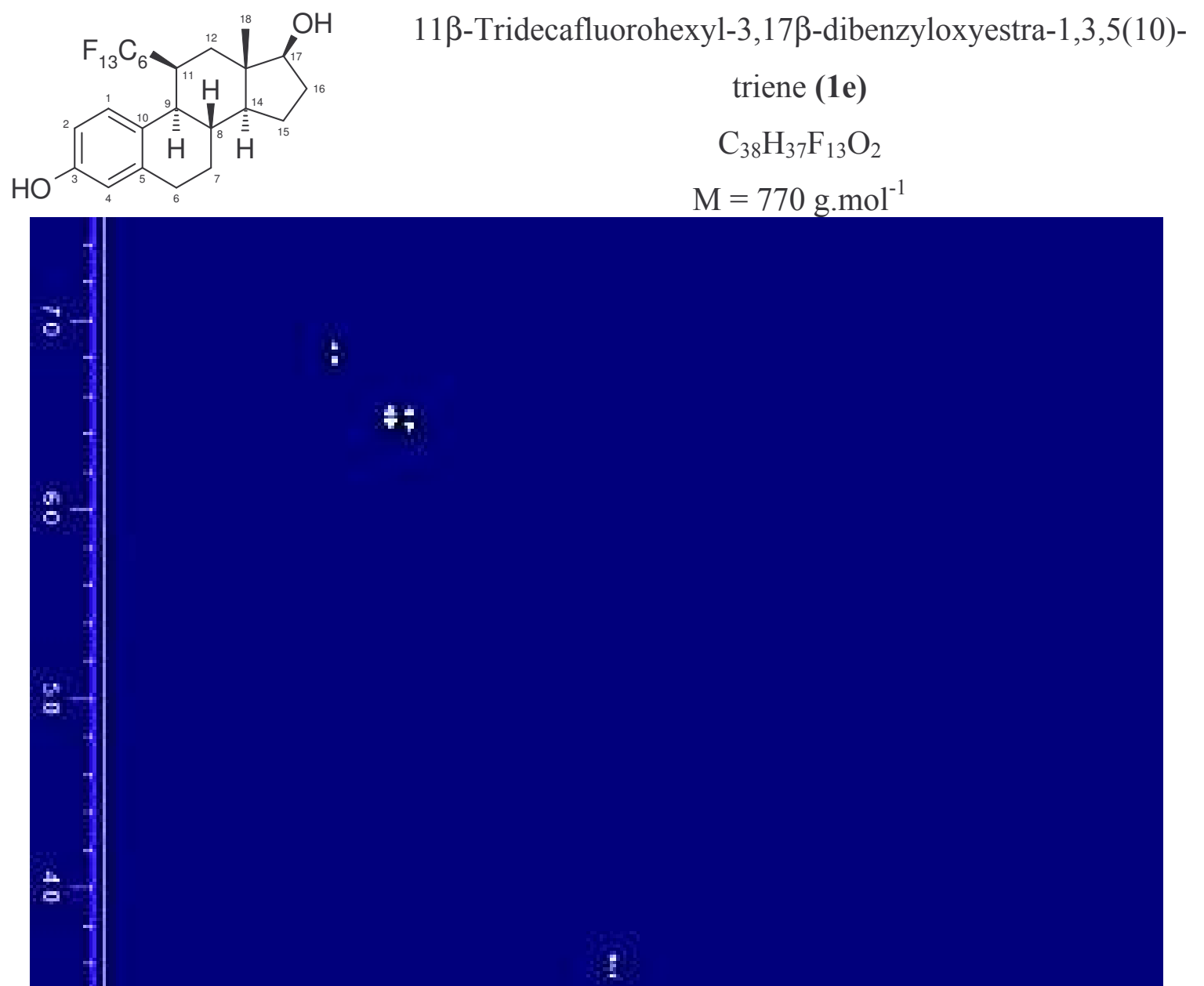

1

8

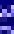

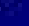


- Compounds 5 and $7: \Delta 8(9)$ or $\Delta 9(11) ?-$

In compound 7, both $\mathrm{H} 12$ show no further coupling with $\mathrm{H} 11$ which is consistent with the putative position of the double bond.

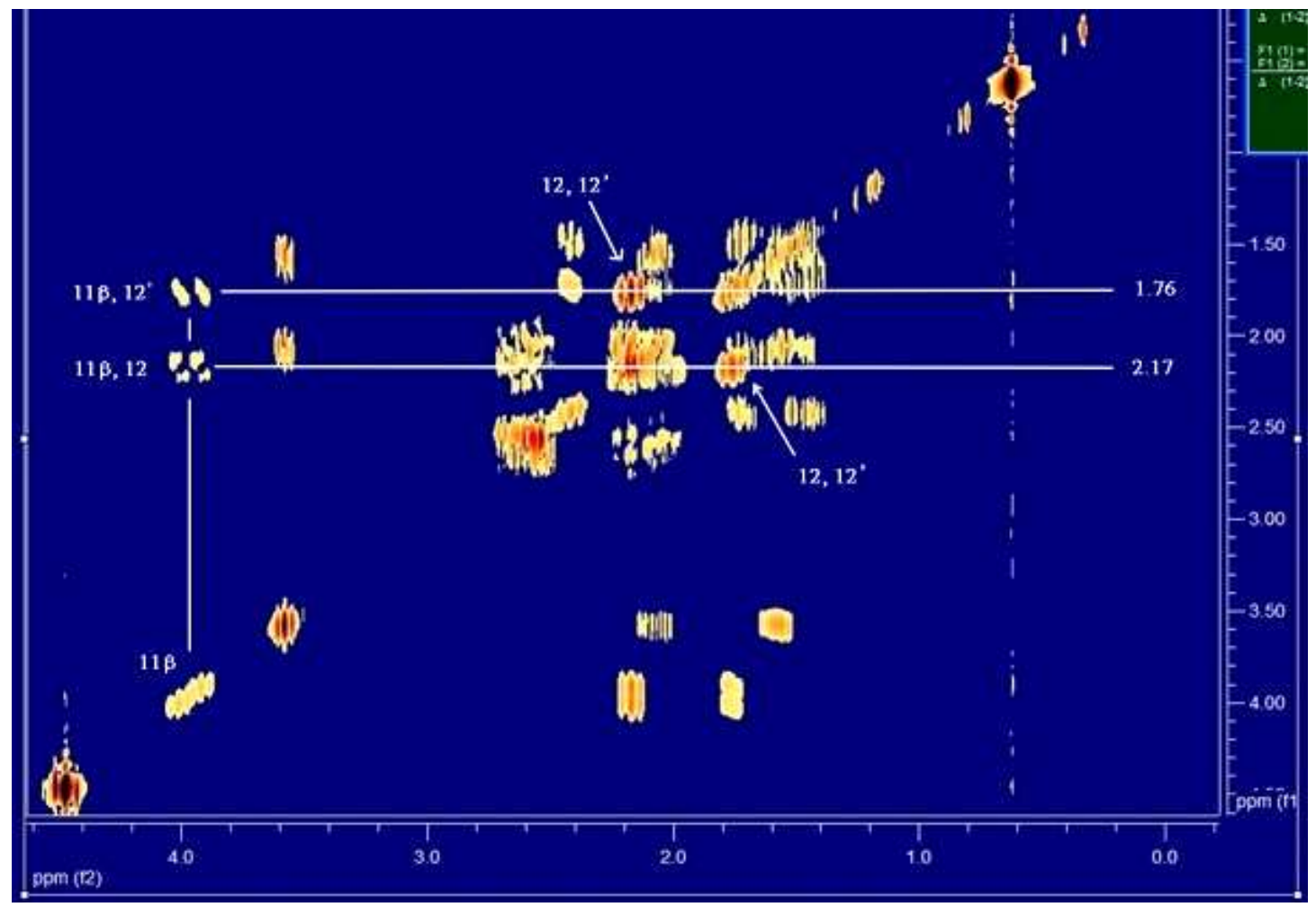

COSY experiment on $\Delta 8(9)-11 \alpha$-perfluorohexyl dibenzyloxyestradiol 5 (above) and $\Delta 9$ (11)-11-perfluorohexyl estradiol 7 (below)

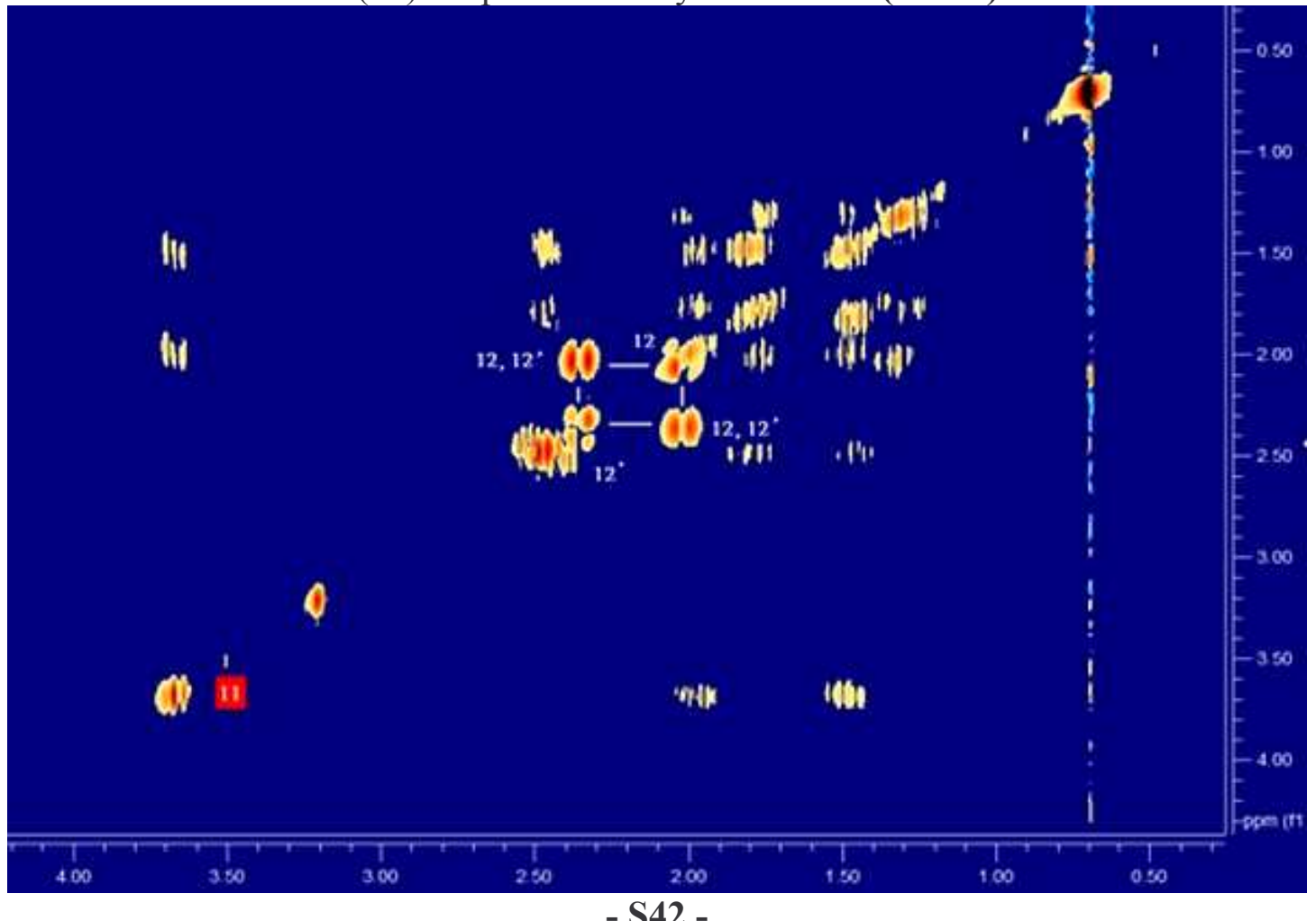

